\title{
How agile teams make self-assignment work: a grounded theory study
}

\author{
Zainab Masood ${ }^{1} \cdot$ Rashina Hoda ${ }^{2} \cdot$ Kelly Blincoe $^{1}$ \\ Published online: 4 September 2020 \\ (C) The Author(s) 2020
}

\begin{abstract}
Self-assignment, a self-directed method of task allocation in which teams and individuals assign and choose work for themselves, is considered one of the hallmark practices of empowered, self-organizing agile teams. Despite all the benefits it promises, agile software teams do not practice it as regularly as other agile practices such as iteration planning and daily stand-ups, indicating that it is likely not an easy and straighforward practice. There has been very little empirical research on self-assignment. This Grounded Theory study explores how self-assignment works in agile projects. We collected data through interviews with 42 participants representing 28 agile teams from 23 software companies and supplemented these interviews with observations. Based on rigorous application of Grounded Theory analysis procedures such as open, axial, and selective coding, we present a comprehensive grounded theory of making self-assignment work that explains the (a) context and (b) causal conditions that give rise to the need for selfassignment, (c) a set of facilitating conditions that mediate how self-assignment may be enabled, (d) a set of constraining conditions that mediate how self-assignment may be constrained and which are overcome by a set of (e) strategies applied by agile teams, which in turn result in (f) a set of consequences, all in an attempt to make the central phenomenon, self-assignment, work. The findings of this study will help agile practitioners and companies understand different aspects of self-assignment and practice it with confidence regularly as a valuable practice. Additionally, it will help teams already practicing self-assignment to apply strategies to overcome the challenges they face on an everyday basis.
\end{abstract}

Keywords Self-assignment - Task allocation agile practice · Agile software development · Grounded theory

Communicated By: Tony Gorschek

Zainab Masood

zmas690@aucklanduni.ac.nz

Extended author information available on the last page of the article 


\section{Introduction}

The success of any software project depends heavily on the execution of the related management activities (Pinto and Slevin 1988). These activities primarily include organizing the software teams, allocating tasks, and monitoring time, budget, and managing resources (Boehm 1991; Jurison 1999) and carried out differently depending on the project management approach followed. In traditional software development, a project manager plays a key role in task allocation (Guide 2001; Nerur et al. 2005; Stylianou and Andreou 2014). The duties of a project manager include planning, assigning, and tracking the work assigned to the project teams. Work is typically allocated keeping in mind the knowledge, skills, expertise, experience, proficiency and technical competence of the team members (Acuna et al. 2006).

In contrast to the traditional development processes, agile software development offers a different approach towards managing the software development cycle particularly task allocation. Instead of the manger assigning the tasks, the team members pick tasks for themselves through self-assignment. This concept of self-assignment is unique to agile software development and emerges from the two principles in the agile manifesto i.e. 'The best architectures, requirements, and designs emerge from self-organizing teams', 'Build projects around motivated individuals. Give them the environment and support they need and trust them to get the job done' (Beck et al. 2001). Even though self-assignment is not directly specified by these principles, but they build the motivation and highlight the significance to study selfassignment.

In theory, agile methods, particularly the Scrum methodology, encourage self-assignment for the allocation of tasks among team members (Hoda et al. 2012; Hoda and Murugesan 2016). Self-directed task allocation or self-assignment is also considered a fundamental characteristic of self-organized teams (Vidgen and Wang 2009; Deemer et al. 2012; Hoda and Murugesan 2016; Strode 2016; Hoda and Noble 2017). Typically, agile methods like XP, Scrum, and Kanban encourage team members to assign tasks or user stories to themselves (Schwaber and Sutherland 2011; Deemer et al. 2012; Hoda and Murugesan 2016). The different agile methods refer to this notion through different terminologies such as selfassigning, signing up and pulling (Beck 2005; Lee 2010; Deemer et al. 2012). We refer to it as self-assignment in this study. Unlike agile practices that have been well-studied such as pair programming (Williams et al. 2000), daily stand-ups (Stray et al. 2016), and retrospectives (Andriyani et al. 2017), it is unclear how self-assignment works in agile projects making it a promising area to study.

In practice, the transition from the manager-led allocation to self-assignment is easier said than done. This transition may not happen in one day due to multiple reasons. The manager may not trust teams and individuals (Hoda and Murugesan 2016; Stray et al. 2018) and resist adopting new ways of working and delegates tasks. The team members may not be comfortable to self-assign tasks themselves due to lack of confidence. Some members may always pick familiar tasks, and others may prefer self-assigning exciting tasks (Vidgen and Wang 2009; Hoda and Murugesan 2016; Strode 2016; Masood et al. 2017b). The team members may self-assign low priority desirable tasks ignoring the high priority ones (Masood et al. 2017b). This indicates that self-assignment can be challenging to practice. The related research does not cover the various aspects of self-assignment in-depth such as comparing the benefits of practicing self-assignment to manager-led allocation, challenges of practicing self-assignment. Additionally, limited information on the strategies agile practitioners follow to overcome the challenges of self-assignment increases the gap in the current research. Therefore, there is a 
need to investigate how self-assignment works in agile teams to answer several open questions such as: What leads to practicing self-assignment? What facilitates self-assignment in agile teams? What constrains self-assignment in agile teams? How do agile practitioners overcome the constraining conditions?

This research is part of a broader study which aims to cover various aspects of selfassignment in multiple phases. As part of our future work, we plan to study various aspects of self-assignment in multiple phases. Some of these aspects are understanding the selfassignment process, motivational factors to self-assigning tasks, role of manager in selfassignment. The focus of this paper is to investigate what leads to practicing self-assignment, conditions influencing the self-assignment process, strategies to overcome the constraining conditions, and any consequences of adopted strategies. It is to be noted that other aspects such as the self-assignment process which includes how and when self-assignment is practiced in agile teams, in what form teams and individual self-assign tasks, and factors individuals keep into account while self-assigning work items are part of the complete doctoral study on selfassignment. Some of the data from phasel of this study has been published (Masood et al. 2017a; Masood et al. 2017b) and reported as preliminary research on self-assignment in related works in this paper (in Section 2 and 5.1).

This study involved 42 participants representing 28 agile teams from 23 software companies based in New Zealand, India, and Pakistan. We collected data in two phases through preinterview questionnaires, semi-structured interviews, and observations of agile practices such as daily stand-ups, iteration planning meetings, and self-assignment during task breakdown sessions. As a result of applying data analysis procedures, we present our grounded theory of making self-assignment work that describes what leads to and facilitates self-assignment, strategies used by the agile teams to make self-assignment work despite constraining conditions, details of the phenomenon of making self-assignment work, along with causal conditions, context, intervening conditions, strategies, and consequences. Additionally, we provided a list of practical implications and recommendations for agile teams, scrum masters and managers practicing self-assignment or teams that are transitioning into self-assignment.

The main contributions of this study are that it illustrates in-depth theoretical knowledge of self-assignment as a task allocation practice in agile teams. Future researchers can refer to this study for understanding the different aspects of self-assignment. Secondly, the practical strategies and recommendations presented in this study will contribute to the software industry by helping managers and agile teams overcome the hurdles and challenges faced in practicing self-assignment.

The remainder of the paper is structured as follows. Section 2 describes related works, section 3 summarizes the research method, sections 4 presents the findings of this research and Section 5 discusses the findings and compares with related work with recommendations for agile community and future researchers. Section 6 concludes the paper.

\section{Related Works}

Software project management comprises of a set of activities which include but are not limited to project planning, scope definition, cost estimation and risk management (Boehm 1991; Jurison 1999). In the conventional process of software development, the activities for project planning such as project schedule, resource and task allocation are taken care of by the project manager (Nerur et al. 2005; Stylianou and Andreou 2014). Resource and task allocation are 
considered important activities in the project planning phase irrespective of what methodology is used in software development. The project manager is considered to be a single point of contact with the sole responsibility of taking the task allocation decisions and managing the project scope and team (Stylianou and Andreou 2014). The project manager role is both critical and challenging as the competence of the project manager and how well they plan and execute these activities significantly contributes to the success of the project. In fact, the managers' decisions on allocating developers and teams to project tasks and scheduling developers and teams are considered one of the key indicators of success of a software project (Stylianou and Andreou 2014).

With the advent of agile software development more than two decades ago, task allocation is no longer the lone responsibility of a manager (Nerur et al. 2005); rather, it is meant to be shared within an empowered development team. Agile introduced light-touch management (Augustine et al. 2005) giving autonomy, empowerment and flexibility to development teams and valuing customers through engagements without forfeiting governance (Beck et al. 2001; Augustine 2005; Carroll and Morris 2015). One of the fundamental characteristics of agile methods is that they support task assignment as a team- and individual-level activity and disregard the traditional role of the project manager w.r.t. tasks delegation (Nerur et al. 2005). Typically, teams practicing agile methods self-assign technical tasks or user stories during the development cycle (Hayata and Han 2011; Hoda and Murugesan 2016). Agile methods are seen to term this self-assignment differently such as "volunteering", "signing up", "committing", and "pulling" (Beck 2005; Lee 2010; Deemer et al. 2012). Empirical studies have been conducted on novice (Almeida et al. 2011; Lin 2013) and experienced agile teams (Masood et al. 2017a; Masood et al. 2017b) to study task allocation decisions, strategies and workflow mechanisms. These studies inform us that tasks assignment in Agile teams is not the sole responsibility of the manager or other team members.

Self-directed task allocation or self-assignment is acknowledged as a fundamental characteristic of self-organized teams (Vidgen and Wang 2009; Deemer et al. 2012; Hoda and Murugesan 2016; Strode 2016; Hoda and Noble 2017). Yet, research on self-assignment in agile software teams has been limited in scale and depth. The focus of such studies has mostly been around task allocation in global software development (Simão Filho et al. 2015). Mak and Kruchten (2006) proposed an approach to address issues that managers face for taskcoordination and allocation in global software development environments using agile methods. The proposed solution and Java/Eclipse-based distributed tool 'NextMove' was meant to facilitate project managers in the prioritization of current tasks and generation of suitability ranking of team members against each available task helping project managers in making dayto-day task allocation decisions. Other researchers have proposed approaches (Mak and Kruchten 2006), models (Almeida et al. 2011) and frameworks (Lin 2013) to address task allocations problems in global software development contexts where agile was being used. The unique context of global software development implies the challenges of task allocation were more to do with the teams being distributed rather than them practicing agile methods.

Self-assignment of tasks has also been observed in open source software (OSS) development in both commercial and non-commercial projects (Crowston et al. 2007; Kalliamvakou et al. 2015). In an empirical study (Crowston et al. 2007), developers' interaction data from three free/libre open source software (FLOSS) projects was examined to understand the process by which developers from self-organized distributed teams contribute to project development. Self-assignment was reported as the most common mechanism among five task assignment mechanisms, the remaining being, (a) assign to a specified person, (b) assign to an 
un-specified person, (c) ask a person outside project development team, and (d) suggest consulting with others. Task allocation in FLOSS development was seen to not involve any micro-management or task delegation through a project manager or an employer. Since these teams are composed of volunteers, the task assignment was mostly based on the personal interests of the contributor. The study identified several drawbacks such as people picking work, they are not good at or lacking prior experience which could impact the quality of the contribution and may require review by others. Similarly, developing code management practices and designing and using such tools is challenging when multiple developers contribute to the same parts of the project.

Existing research on self-assignment in co-located, e.g. non-distributed and non-open source, agile teams is very limited. Self-assignment in new agile teams is seen to happen as a gradual process, retaining a manager's role at the beginning for tasks delegation (Hoda and Noble 2017). Our preliminary work conducted on a dataset of 12 agile practitioners from four teams of a single company based in India confirmed five main types of task allocation approaches in agile teams: manager-driven, manager-assisted, team-driven, team-assisted, and or self-directed (Masood et al. 2017a). With time and experience, agile teams seem to dispose of the command and control attitude and are instead seen to move towards managerassisted or team-assisted assignment and, in some cases, towards practicing self-assignment over time (Hoda and Noble 2017; Masood et al. 2017a). As a part of that preliminary work, we also identified some motivational factors that agile developers take into account while selfassigning tasks such as technical complexity, business priority, previous experience with similar tasks, and others (Masood et al. 2017b). However, we do not know in-depth what strategies the teams use to make self-assignment work despite certain intervening conditions. In this study, we investigated how self-assignment works in agile teams in a way that it's not only beneficial to individuals, teams, and projects but also to the organizations.

Here we presented an overview of the related works of task allocation in agile software development. We will revisit them in light of our findings in Section 5, comparison to related work.

\section{Research Method}

After considering a number of potentially suitable methodologies such as Case study (Yin 2002), Ethnography (Fetterman 2019), and Grounded Theory (Glaser 1978; Strauss and Corbin 1990), we adopted Grounded Theory (GT). The interest of researchers towards generating a theory to explain how agile teams make self-assignment work using a crosssectional dataset not limited to few cases or organizations led the researchers to use GT. The intention is to uncover self-assignment from empirical data rather than validating any existing theories or hypotheses. Also, the focus of this study is around understanding the process, investigating strategies, and exploring underlying behaviours, and influencing factors, and so GT was particularly well-suited.

GT comes in various versions, Classical/Glaserian, Strauss and Corbin, and Charmaz Constructivist, we employed the Strauss and Corbin version due to several reasons:

a. It follows a more prescriptive approach than classical GT (Coleman and O'Connor 2007; Kelle 2007) leading the researcher through clear guidelines, and, as a novice GT researcher, the first author found this useful. 
b. It builds on research question which is open ended and drives the direction of research (Strauss and Corbin 1998).

c. It provides an additional analytic tool for axial coding in the form of a coding paradigm, which can help GT researchers identify the categories, sub-categories, and their relationships much earlier in contrast to classical GT theory where this emerges after multiple rounds of analysis (Seidel and Urquhart 2016).

The study comprises of two phases, each including multiple iterations of data collection and analysis as shown in Fig. 1. In the first phase, we explored the task allocation process in Agile teams. In the second phase, we narrowed down our focus to self-assignment as a specific task allocation process. We collected the data in multiple rounds, data of each round was analysed before collecting more data to ensure theoretical sampling. This was done until we reached theoretical saturation. This is evident from our interview questions which were revisited and revised to meet the narrowing focus of the GT study. The primary data sources for phase1 were face-to-face interviews and for phase 2 were pre-interview questionnaires, face-to-face semistructured interviews, and team observations of agile practices. We describe these in the following sections. The additional documents, such as interview guides, pre-interview questionnaire etc. can be found as supplementary material (Masood et al. 2020).

\subsection{Data Collection \& Analysis (Phase1)}

Phase1 aimed to investigate the task allocation process in agile teams. The focus was to study the task allocation strategies in agile teams. The authors collectively prepared the interview guide (all authors), conducted semi-structured interviews (second author), transcribed the

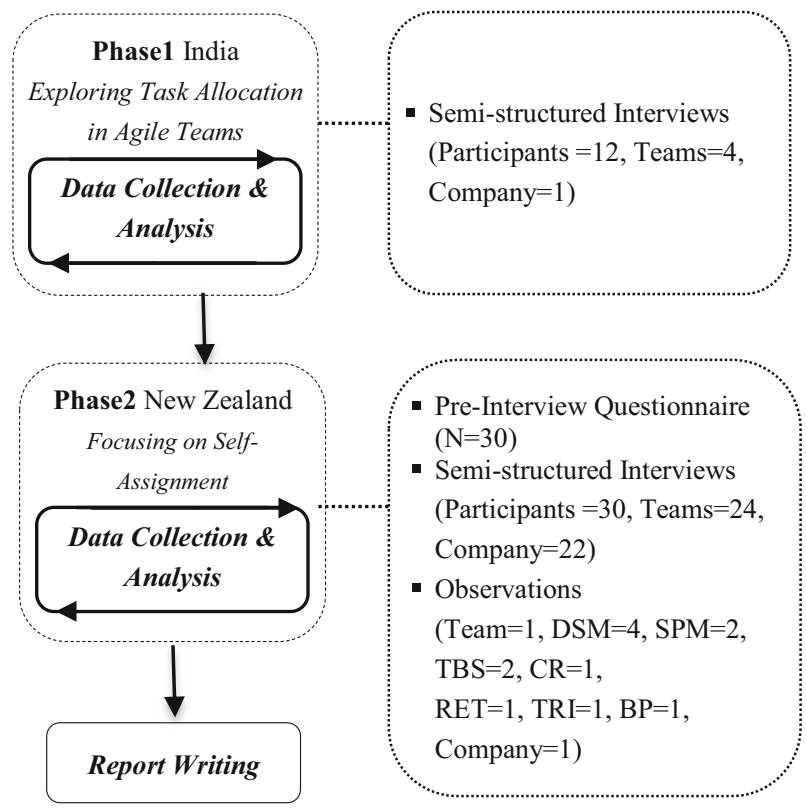

Fig. 1 Phases of iterative Data Collection \& Analysis (DSM = Daily Stand-Up; SPM = Sprint Planning Meeting; TBS = Task Breakdown Session; $\mathrm{CR}=$ Code-Review; RET $=$ Retrospective; TRI=Squad Triage; $\mathrm{BP}=\mathrm{Backlog}$ Prioritization) 
interview recordings (first author) and analysed (all authors) to reduce any bias and improve internal validity through researcher triangulation. The interview guide designed to collect data for this phase focused on four main areas (Fig. 2):

a. professional background: e.g. please tell me about your professional background

b. agile experience e.g. how long have you been using agile practices?

c. current team and project, e.g. which practices have been used regularly on this project?

d. task allocation practices e.g. how does task allocation happen in your team?

We sent invitation to the "Agile India" group to recruit participants for phase1. An Indian software company responded with a willingness to participate in the study. We interviewed 12 participants in-person from that company. Table 1 summarizes the demographics of the participants (P1-P12), highlighted in lighter shade of grey. Each interview took approximately 30-60 min. These face-to-face interviews helped to record the verbal information and capture the interviewee's expressions and tone (Hoda et al. 2012). All these interviews were recorded and transcribed for analysis. The data collected from phase1 was manually added in NVivo data analysis software. The data collected helped in developing an initial understanding of task allocation in agile teams. We applied open coding, the Strauss and Corbin GT's procedure of data analysis (Strauss and Corbin 1990) on participants' transcribed interview responses. During open coding, we labelled the data with short phrases that summarize the main key points. These were further condensed into two to three words, captured as codes in the NVivo. As a result of data analysis, different concepts from similar codes emerged, one the most prominent of which was task allocation through self-assignment. Others included managerdriven, manager-assisted, team-driven, and team-assisted task allocation (Masood et al. 2017a). The results of phase 1 directed us to focus on self-assignment as the substantive area of the study in the next phase.

\subsection{Data Collection (phase 2)}

Phase2 aimed to investigate self-assignment as a task allocation practice and explore how agile teams make self-assignment work. The goal of the study was to build a theory to identify what leads to and facilitates self-assignment process, what strategies are used by the agile teams to make self-assignment work, and the consequences of these strategies. As with phase1, the authors collectively prepared the instruments i.e. pre-interview questionnaire and interview guide (all authors), conducted interviews (first author), and analysed them (all authors) to mitigate potential bias. The pre-interview questionnaire gathered basic and professional details of the participants and the interview guide was primarily used to facilitate the interviewer and the interview process to collect details around various aspects of self-assignment. The interview guide was refined throughout to accommodate the exploratory nature of the study. All the interviews conducted during phase 2 were transcribed for analysis either by the first author or the third-party transcribers. The pre-interview questionnaire and the interview guide used to collect data during the phase2 focused on the following main areas (Fig. 2):

a. professional background: e.g. please tell me about your professional background
b. agile experience e.g. how long have you been using agile practices?
c. current team and project, e.g. which agile practices have been used regularly on this project? 


\section{Phase 1: Interview Guide}

Sample demographic type questions

...............

Q. Total experience in software industry
Q. Total agile experience (years) Years

Sample open-ended semi-structured questions

Q. How does task allocation happen in your team?

Q. In what form does work arrive to your team? Q. In what form does work arrive to you (individually)?

Q. Who allocates work to your team? Q. When does individual task allocation happen?

Phase 2: Pre-Interview Questionnaire

Sample demographic type questions

Q. Total experience in software industry
Q. Total agile experience (years) Years

Q. Current Company Name

Q. Reference to company e.g. website

Q. Type of company Check all that apply. Single Product/ Multiple Products / Consultancy/ Others (specify name):

Q. Job Title (select all that apply) Check all that apply. Developer/Tester/Scrum Master/Manager /Business

Analyst Other: (specify name):

Sample project and team type questions

Q. Domain of project Check all that apply. IT Finance \& Banking /Transport /Medical / Telecom / Healthcare / Manufacturing Other: (please specify name):

Q. Project focus: Check all that apply. Migration, New Development, Software as a service (SAAS), Other: (please specify)

Q. Team Size (Number of people)

Q. Your role on project

Sample agile practices type questions

Q. How long is the team following agile practices Years?

Q. Agile method used: Check all that apply. None/ Scrum / XP / Combo / Kanban/ Crystal /Other:

Q. Iteration length: Not applicable/ 1 week/ 2 weeks/ 3 weeks / 4 weeks

\section{Phase 2: Interview Guide}

Sample open-ended semi-structured questions

Q. What are your major responsibilities as per [role mentioned in the pre-interview form]?

Q. In your opinion, is it better to pick up tasks for yourself or for tasks to be assigned by a manager? How?

Q. In what form does work arrive to your team? Can you please elaborate with few examples?

Q. Who allocates work to your team and How? Q. In what form do you pick the work?

Q. How does self-assignment takes place in your team?

Q. When do you pick/self-assign tasks?

Q. What problems do you (as a developer)/your team (as a manager) face while picking up tasks?

Q. How do you overcome these problems? Please provide examples with how these problems were solved.

Q. What benefits of self-assignment have you experienced? Please provide some examples.

Q. Based upon your experience, is self-assignment better than delegation? How? Any weaknesses?

Q. Any improvements you would suggest improving current process of self-assignment?

Q. When did your team start with self-assignment? How and when self-assignment was introduced as a practice in your teams?

Q. Based on your experience, what leads to an effective self-assignment?

Q. What are the situations when self-assignment does not take place? Why?

Fig. 2 Examples of interview questions for Phase1 and 2

d. Various aspects of self-assignment, e.g. How does self-assignment take place in your team? What problems do you (as a developer)/your team (as a manager) face while picking up tasks? Please provide an example with how these problems were solved. 
Table 1 Participants Demographics

\begin{tabular}{|c|c|c|c|c|c|c|c|}
\hline $\mathbf{P} \#$ & Team\# & Role & Domain & TX & $\mathbf{A X}$ & $\mathbf{A M}$ & AG \\
\hline $\mathrm{P} 1$ & $\mathrm{~T} 1$ & Tech lead & IT & 11 & $6-7$ & $\mathrm{SC}$ & $31-35$ \\
\hline $\mathrm{P} 2$ & $\mathrm{~T} 1$ & Software engineer & IT & $2-2.5$ & 1 & $\mathrm{SC}$ & $21-25$ \\
\hline P3 & $\mathrm{T} 1$ & Assoc. tech lead & IT & $4-5$ & $4-5$ & $\mathrm{SC}$ & $26-30$ \\
\hline P5 & $\mathrm{T} 2$ & Tech lead & IT; AN & 7 & 7 & $\mathrm{SC}$ & $36-40$ \\
\hline P6 & $\mathrm{T} 2$ & Senior software engineer & IT; AN & 4 & 2 & SC & $26-30$ \\
\hline P7 & $\mathrm{T} 2$ & Tech lead & IT; AN & 7.5 & 7.5 & SC & $31-35$ \\
\hline $\mathrm{P} 10$ & $\mathrm{~T} 3$ & Senior software engineer & IT; CS & 3.5 & 1 & $\mathrm{KN}$ & $21-25$ \\
\hline P11 & T3 & Assoc. tech lead & IT; CS & 4.5 & 2 & $\mathrm{KN}$ & $26-30$ \\
\hline $\mathrm{P} 12$ & $\mathrm{~T} 4$ & Senior software engineer & $\mathrm{HC}$ & 3.5 & 3.5 & $\mathrm{SC} ; \mathrm{KN}$ & $21-25$ \\
\hline P13 & T5 & Consultant & IT & 10 & 3 & SC & $31-35$ \\
\hline P14 & T6 & Tech lead; Developer & MD & 13 & 7 & SC & $31-35$ \\
\hline P 15 & $\mathrm{~T} 7$ & Developer; Scrum Master & TP & 17 & 7 & SC & $36-40$ \\
\hline $\mathrm{P} 21$ & $\mathrm{~T} 11$ & Scrum Master & CRM & 9 & 6 & SC & $36-40$ \\
\hline $\mathrm{P} 22$ & $\mathrm{~T} 11$ & Developer & $\mathrm{HC}$ & 12.5 & 6 & SC & $41-45$ \\
\hline P23 & $\mathrm{T} 12$ & Test Analyst & $\mathrm{FN}$; BK & 10 & 5 & SC & $31-35$ \\
\hline $\mathrm{P} 24$ & $\mathrm{~T} 13$ & Tester & MD & 12 & 1 & $\mathrm{SC} ; \mathrm{KN}$ & $31-35$ \\
\hline $\mathrm{P} 25$ & T14 & Developer & $\mathrm{HC} ; \mathrm{BK}$ & 10.5 & 4 & $\mathrm{SC} ; \mathrm{SP} ; \mathrm{KN}$ & $31-35$ \\
\hline P26 & $\mathrm{T} 15$ & Product Owner & IT; TC; & 12 & 5 & $\mathrm{SC}$ & $31-35$ \\
\hline P27 & $\mathrm{T} 11$ & Developer & $\mathrm{HC}$ & 12 & 2 & $\mathrm{SC} ; \mathrm{KN}$ & $36-40$ \\
\hline P28 & T16 & Developer & IT & 4 & 3.5 & $\mathrm{SC}$ & $26-30$ \\
\hline P29 & $\mathrm{T} 17$ & Developer; Scrum Master & IT & 8 & 3.5 & $\mathrm{SC} ; \mathrm{KN}$ & $31-35$ \\
\hline P30 & $\mathrm{T} 18$ & Lead Developer & IT & 25 & 9 & SC & $46-50$ \\
\hline P31 & $\mathrm{T} 11$ & Development Manager & HR & 20 & 2 & SC & $46-50$ \\
\hline P32 & $\mathrm{T} 19$ & Developer & IT & 12 & 7 & $\mathrm{SC} ; \mathrm{KN}$ & $36-40$ \\
\hline $\mathrm{P} 42$ & $\mathrm{~T} 28$ & Technical Lead & IT; CRM & 14 & 9 & SC & $31-35$ \\
\hline
\end{tabular}

Following Grounded Theory's guidelines of refinement and constant narrowing-down, the interview focused on self-assignment and its various aspects. From phase1, we noticed that capturing participants' demographics data was taking a significant amount of time during the interview, sometimes leaving interesting aspects unexplored. So, for phase2, demographics and supporting details such as professional background, agile experience, current team and project related details were gathered using a pre-interview questionnaire filled by each participant before their interview.

To recruit participants for phase2, we sent invitations to multiple online groups, and those who showed willingness to participate verbally or through emails were contacted. Social networking sites such as LinkedIn, Meetups groups such as "Agile Auckland", "Auckland Software Craftsmanship" served as useful platforms to recruit participants in New Zealand. Once a participant contacted us showing their willingness, we requested them to share basic and professional details through the pre-interview questionnaire. The details gathered from the pre-interview questionnaire also helped us limit our context to individuals and teams who practice self-assignment at some level and with varied frequency (always, frequently, rarely, and occasionally). Agile teams not practicing self-assignment were out of scope. We conducted 30 more interviews ( 28 in-person and 2 via Skype). These semi-structured interviews were 
conducted for 30-60 min per participant. Table 1 summarizes the demographics of the participants involved in the phase 2 of study in darker shade of grey.

The first author attended multiple sessions of agile practices while observing agile team 'T11' comprised of 7 members. This included attending four daily stand-ups of duration 1015 min each, two one-hour sprint planning meetings for two sprints, two-hours task-breakdown sessions for two sprints, one 30-mins code-review session, four squad triage sessions of 10-15 min each which focused on the outstanding issues requiring clarifications, discussions or any decisions, one backlog prioritization $30 \mathrm{mins}$, and an hour long retrospective meeting. Figure 3. captures some glimpses of the sessions attended during these observations. Observations of practices supplemented our understanding of the self-assignment process, practices, and strategies followed by the teams.

The entire study involved 42 participants represented through numbers P1 to P42 for confidentiality reasons. Table 1 summarizes the demographics of all the participants. Participants were working for software companies developing software solutions for healthcare, accounting, finance, transport, business analytics, and cloud services. Participants were working in New Zealand (71.5\%) and India (28.5\%) and varied in gender with $86 \%$ male and $14 \%$ female. Age and professional experience varied from 2 to 25 years of experience. They were directly involved in the software development with job titles as developer, consultant, product owner, architect, lead developer, and scrum master. Most of the participants were practicing Scrum, whereas some used a combination of Scrum and Kanban. They used agile practices such as daily meetings, customer demos, pair programming, iteration planning, release planning, reviews and retrospectives.
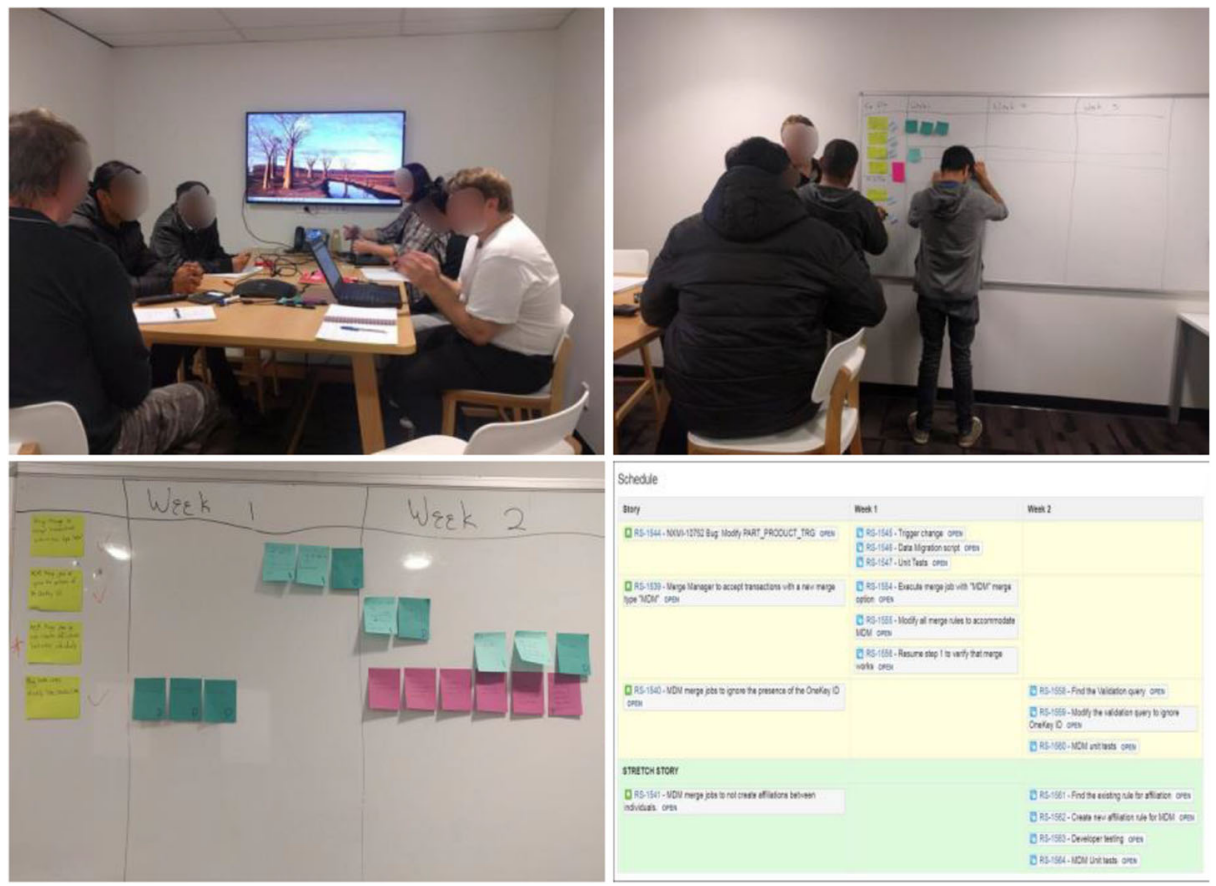

Fig. 3 Team T11 Observations (Top left: Sprint Planning, Top right: Task Breakdown \& Allocation Session, Bottom left: Physical Task Board, Bottom right: Digital Task Board) 


\subsection{Data Analysis (phase 2)}

The Strauss and Corbin's version of GT comprises of three data analysis procedures: open, axial, and selective coding (Strauss and Corbin 1990). All these procedures were interwoven and were conducted mainly by the first author with the underlying steps such as defining emerging codes, concepts, sub-categories, and categories being thoroughly discussed on an on-going basis, and finalized with the co-authors, including a GT expert. The use of analytical tools such as diagramming, whiteboarding, and memo writing facilitated the analysis process. The quantitative data was collected using a pre-interview questionnaire and the qualitative data in the form of transcripts, observation notes, and images were uploaded in NVivo. Figure 4 provides a step-by-step example of applying all these procedures.

Open coding We started the data analysis with open coding, in which all the interview transcripts were analysed either line by line or paragraph by paragraph as appropriate and represented with short phrases as codes in the NVivo software. With constant comparison within same and across different transcripts, we grouped similar codes to define a concept, a higher level of abstraction. Sometimes, multiple concepts were generated from single quotes as shown in a few examples in Fig. 4. These concepts were identified in the data and sometimes defined in terms of their properties and dimensions to contextualize and refine the concepts. The extent to which this could be done relied on the level of details were shared by the participants. Then, we integrated concepts into the next level of data abstraction, categories. The outcome of open coding was a set of concepts and categories.

Figure 4 illustrates the open coding and constant comparison procedures using multiple examples, starting from the raw interview transcripts of the participants [P13, P18, P21, P26, $\mathrm{P} 31]$, and observation notes [T11] listing the category, concept, property and dimensions for each transcript excerpt as examples. For example, excerpt from P13 resulted in multiple

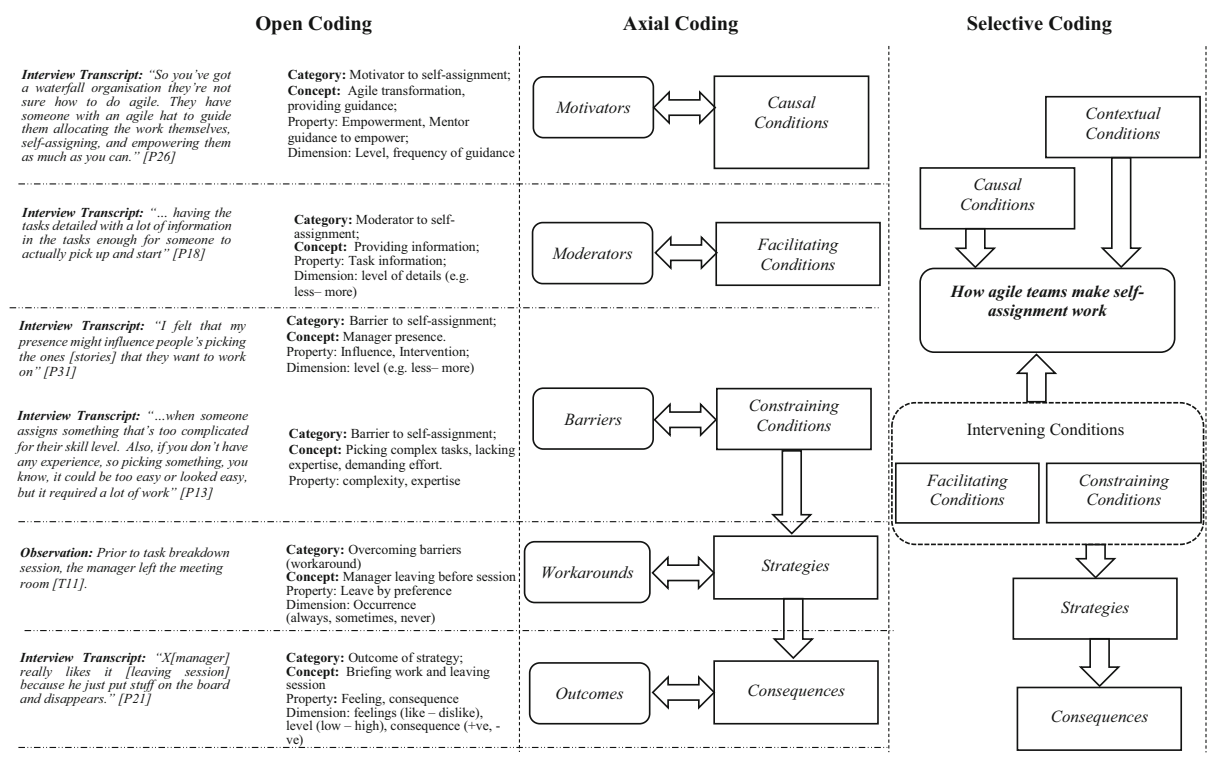

Fig. 4 Example of applying Grounded Theory data analysis procedures, Open Coding, Axial Coding, Selective Coding 
concepts 'picking complex tasks', 'lacking expertise', 'demanding effort'. All these were grouped under the category 'barriers to self-assignment'. These came from the answers to questions like 'What problems and challenges do you (as a developer)/your team (as a manager) face while picking up tasks?'. In addition to concepts and categories, we also identified properties and dimensions. Properties are 'characteristics that define and explain a concept' and dimensions are 'variations within properties'. For example, one of the participants P31 shared that their presence influenced people's self- assignment choices and decisions. This led us to classify 'intervention' as a property, and 'intervention level' as a dimension (see Fig. 4). The open coding process was applied on the entire data set (interviews and observations) of the study. This way all the conditions, strategies and consequences were identified, categorized, and reported. The categorisation was discussed during regular team meetings and refined with constant feedback from the co-authors.

Axial coding Next, we applied axial coding, a 'process of systematically relating categories and sub-categories'. Sub-categories are also concepts that refer to a category providing further clarifications/details. Strauss recommends using 'analytical tools' to define relationships between categories and sub-categories (Strauss and Corbin 1990). One such tool is Coding Paradigm which guides the researcher to illuminate the conceptual relationships between concepts/categories by identifying the conditions, actions/interactions, and consequences associated with a phenomenon. Strauss proposed variants of the coding paradigm to facilitate axial coding (Urquhart 2012). All of these are used as analytical tools and organization schemes (Corbin and Strauss 2008) which help to arrange the emerging connections and identify the relationships. To the best of our knowledge, this is one of the very few software engineering research studies (Giardino et al. 2015; Lee and Kang 2016) that apply and illustrate an in-depth application of Strauss and Corbin's Grounded Theory, including the use of their "coding paradigm" (in Fig. 5, presenting the Phenomenon, Context, Causal Condition, Intervening Conditions, Strategies, and Consequences).

We applied the coding paradigm as it assembled our data well, the structure of the coding paradigm mapped well to our emerging categories (Strauss and Corbin 1990; Corbin and Strauss 2008). The coding paradigm contains different terminologies such as context, causal conditions, intervening conditions, action/interactional strategies, and consequences. Table 2. states the Strauss and Corbin definitions of the coding paradigm terminologies ( 2 nd column) with how we applied them to our study on self-assignment ( 3 rd column). Our categories from open coding such as motivators, barriers, moderators, outcomes are mapped to the coding paradigm terminologies such as causal conditions, intervening conditions, consequences (represented by the symbol $\Leftrightarrow$ in Fig. 4). The relationships between the sub-categories (other categories) were represented as per the terminologies of the coding paradigm (defined in Table 2, represented by $\Rightarrow$ in Fig. 4). For instance, we know from the definition that causal conditions are the reasons why teams adopt self-assignment and leads to the phenomenon under study. Similarly, we know that the strategies to work around challenges of self-assignment result in different consequences. All the relationships between categories and sub-categories were iteratively and constantly validated with the data (within each participant's data and across other participants' data) throughout the analysis process. This was done in multiple analytical iterations and the relational statements evolved with continuous reflections over time.

In Selective Coding, we started building a storyline presenting the essence of our study where each sub-category and category captured a part of the whole story of making selfassignment work (presented in Fig. 4). How agile teams make self-assignment work emerged 


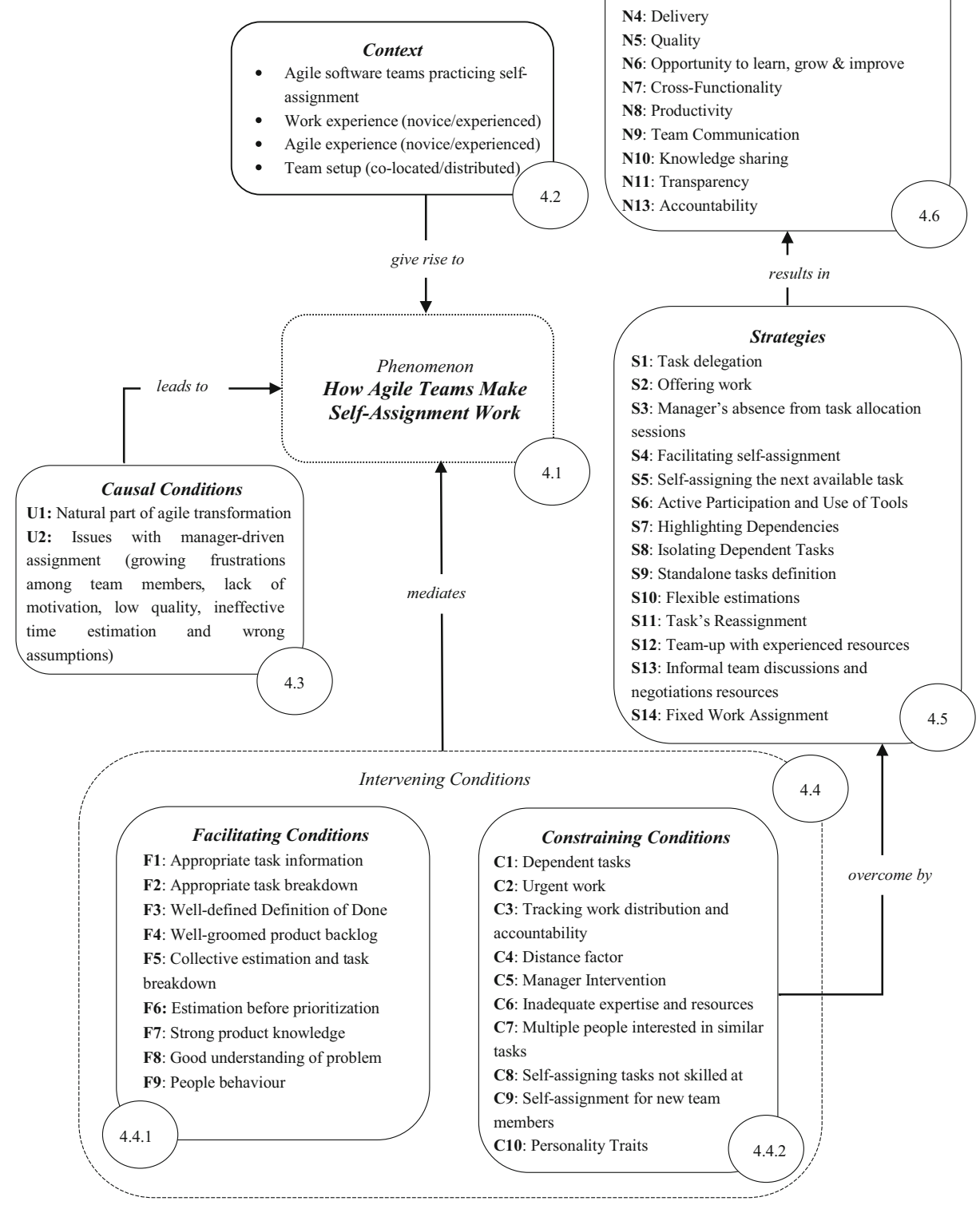

Fig. 5 How agile teams make self-assignment work (using Strauss's Coding Paradigm, including Phenomenon, Context, Causal Condition, Intervening Conditions, Strategies, and Consequences)

as the most prominent and central phenomenon from our data analysis process (described in section 4) that was binding all the sub-categories together, strengthening the relationships identified during the axial coding. It was during the selective coding, we confirmed which relational phrases such as 'mediates', 'overcome by', 'give rise to' were fitting well to our 
Table 2 Terminologies of Coding Paradigm (Strauss and Corbin 1990) as applied in this study

\begin{tabular}{|c|c|c|}
\hline Terminologies & Strauss and Corbin Definition & Our study \\
\hline Phenomenon & $\begin{array}{l}\text { The actions of an individual as well as } \\
\text { interactions between different people revolve } \\
\text { around the phenomenon }\end{array}$ & $\begin{array}{l}\text { Making self-assignment work represents } \\
\text { the phenomenon }\end{array}$ \\
\hline Causal Conditions & $\begin{array}{l}\text { Events, incidents, happenings that lead to the } \\
\text { occurrence or development of a } \\
\text { phenomenon }\end{array}$ & $\begin{array}{l}\text { Causes leading to self-assignment, } \\
\text { reasons why teams adopt } \\
\text { self-assignment }\end{array}$ \\
\hline $\begin{array}{l}\text { Intervening } \\
\quad \text { Conditions }\end{array}$ & $\begin{array}{l}\text { The conditions that intervene the strategies } \\
\text { taken within a specific context }\end{array}$ & $\begin{array}{l}\text { Facilitating and constraining conditions } \\
\text { influencing self-assignment }\end{array}$ \\
\hline $\begin{array}{l}\text { Context/Contextual } \\
\text { Conditions }\end{array}$ & $\begin{array}{l}\text { Context represents the particular set of } \\
\text { conditions within which the } \\
\text { action/interactional strategies are taken }\end{array}$ & $\begin{array}{l}\text { The specific set of conditions within } \\
\text { which the strategies to make } \\
\text { self-assignment work occur }\end{array}$ \\
\hline $\begin{array}{l}\text { Action/Interactional } \\
\text { Strategies }\end{array}$ & $\begin{array}{l}\text { Strategies devised to manage, handle, carry out, } \\
\text { and respond to a phenomenon under a } \\
\text { specific set of perceived conditions }\end{array}$ & $\begin{array}{l}\text { Adopted strategies to work around } \\
\text { challenges of self-assignment }\end{array}$ \\
\hline Consequences & Outcomes or results of action and interaction & $\begin{array}{l}\text { Impact of adopting these strategies to } \\
\text { make self-assignment work }\end{array}$ \\
\hline
\end{tabular}

entire theory model in Fig. 5. It was also during the selective coding, when theoretical saturation was reached and no new concepts, categories or insights were identified. Then, finally we revisited and refined the categories to make sense of the entire theory explaining the phenomenon.

\section{Results}

We present our grounded theory of making self-assignment work in agile teams. The section is structured to follow Fig. 5. which visually represents our theory and illustrates its categories in the following sub-sections in detail. In the following sections, we present all our findings that comprise the overall theory (Fig. 5), including plenty of quotations from the raw data and sample observation notes/memos.

The grounded theory of making self-assignment work in agile teams explains the (a) context (described in section 4.2) and (b) causal conditions that give rise to the need for selfassignment (described in section 4.3), (c) a set of facilitating conditions that mediate how self-assignment may be enabled (described in section 4.4.1), (d) a set of constraining conditions that mediate how self-assignment may be constrained (described in section 4.4.2) and which are overcome by a set of (e) strategies applied by agile teams (described in section 4.5), which in turn result in (f) a set of consequences (described in section 4.6), all in an attempt to make the central phenomenon, self-assignment, work.

\subsection{The Phenomenon - How Agile Teams Make Self-assignment Work}

One of the key findings of our study is that self-assignment is not as easy and straightforward as might be expected. It comes with challenges and requires a set of strategies to make it work in practice. Our findings indicate clearly that self-assignment does not simply imply picking whatever tasks team members want. Development team members are bound to choose tasks based on their business needs and priorities as stated by P30. 
'It's not just like, go out there and choose whatever you want to work on...it's like team commits, and whatever they've committed, they've selected tasks from a triaged [prioritized] list and they're committing to that work.' - P30, Lead Developer

We identified that transitions to self-assignment does not happen automatically but teams with a positive mindset, an encouraging Scrum Master who values teams and empowers autonomy, and the use of effective strategies lead to effective self-assignment smoothly. As such, the key phenomenon identified in our analysis was "how agile teams make self-assignment work".

\subsection{The Context- Contextual Details and Conditions}

Beyond the demographics captured in the pre-interview questionnaires (participant age, gender, experience, etc.), other contextual details emerged during our in-person interviews and while observing team practices to understand how self-assignment works. The variation in the team setup (co-located, distributed), work experience (novice, experienced) and team's agile experience (novice, transitional, mature) can have influence on the facilitating/constraining conditions and corresponding strategies. We will see that the contextual conditions vary in their application. For example, strategies identified to facilitate self-assignment in distributed team contexts were different to those for co-located team context. Similarly, strategies for new team members were different from those for mature, experienced teams. While manager intervention may not be a constraining condition for teams with flat structure without managers and so the strategies cannot be applied in such settings. Teams self-selecting their tasks at the beginning of the sprint may have different constraining conditions when compared to teams which self-assign the tasks during the sprint. The contextual details are best understood in relation to the related conditions and strategies, and so these contextual details are weaved into our descriptions in the following sub-sections.

\subsection{The Causal Conditions - Leading to Adopting Self-assignment}

In this study, the participants were questioned about why they chose to self-assign. In result, we identified many different reasons for adopting self-assignment. The most common cause was it being a natural part of the agile transformation represented as U1. Other causes reported by the participants are related to issues with manager-driven assignment referred by U2. We used the term 'manager' to refer to all management roles (i.e. project managers, scrum masters, and team leads).

\subsubsection{U1: Natural Part of Agile Transformation}

The most common rationale $[N=10]$ behind opting to practice self-assignment evolved naturally with an understanding of the scrum methodology (Deemer et al. 2012) and agile manifesto (Beck et al. 2001). As teams adopted agile methods, they also became more selforganized.

‘...It [self-assignment] naturally started off that individuals in a team are responsible to go and select ... So, I think it was just our understanding of the Scrum methodology and agile Manifesto' -P42, Technical Lead 


\subsubsection{U2: Issues with Manager-driven Assignment}

Issues with the manager-driven assignment approach caused some participants to drift towards self-assignment. These issues include growing frustrations among team members, lack of motivation, low quality of work and inaccurate estimates.

Growing frustrations among team members A quality assurance analyst P36 identified frustrations as a cause that led to the team adopting self-assignment. The Scrum Master may not always be aware of frustrations of the team, as explained by the participant, recalling a particularly challenging experience:

'It was one Quality Assurance Analyst, she broke down, saying that I can't do it anymore. She was required [assigned] to test something in the cloud, introducing her in just the last minute... When I saw her collapsing down, I had lots of empathy with her. And then in our retrospective, I also started exploding and I'm not taking any allocation. This is all going wrong. The scrum master went back, she came again, and she said, I will not allocate anything, you, as a team, sort out the distribution.'-P36, Quality Assurance Analyst

Lack of motivation Some participants described that team members are more motivated and happier when they have some level of ownership and when they see value in what they're doing. For example, participant P41 highlighted lack of motivation as a reason to replace the manager-driven task allocation with self-assignment and participant P40 revealed happiness among team members with self-assignment.

'Prior to this [self-assignment] they [team members] were less motivated' -P41, Senior Architect

'With self-assignment people are happier. They feel more in charge of what they're doing, they have that sense of ownership.'-P40, Consultant

Low quality It was also indicated that when it was someone else in the team assigning the tasks, the quality of the work was not that good. This could be because the person assigning the task may not always be well-aware of an individual's technical skills and interests.

'[Earlier] most of the time it was Scrum Master or the PM's say who's going to do what.... and the quality of the output wasn't that great' - P37, Head of Product Delivery

This in a way is correlated with lack of motivation as work quality is good when the team members are motivated and more committed.

'When they [team members] are motivated, I see them delivering exceptional results' P41, Senior Architect

Inaccurate estimates It was also reported that the shortcomings of manager-driven task allocation helped participants take up self-assignment. One of these shortcomings was the possibility of making wrong assumptions because the manager was not always fully aware of the actual implementation details, underlying technical risks, and the expected time to perform a task, potentially leading to inaccurate estimates. 
'When a manager hands it [user stories/tasks] down, often they'll either make estimates, and then they'll hold you to their estimates and then there are all sorts of problems. P15, Technical Lead \& Scrum Master

The developer P16, agreed with the Scrum Master's point of view.

'Team deciding on their own capacity is better than being handed down [estimates] because if a manager puts their finger in the air and makes a wrong assumption, that sends unrealistic message to the business' - P16, Developer

\subsection{The Intervening Conditions - Conditions Influencing Self-assignment}

These causal conditions led agile teams to adopt and practice self-assignment. Next, we will see what and how the intervening conditions influence the self-assignment process. We have elaborated these conditions as factors that facilitate or constrain our phenomenon. The conditions that facilitated the self-assignment process are described as facilitating conditions in sub-section 4.4.1 and the conditions that hindered the process are mentioned as constraining conditions in sub-section 4.4.2. These are listed in Table 3.

\subsubsection{Conditions Facilitating Self-assignment}

There are certain facilitating conditions, which are broad, general conditions that influence the phenomenon. The phenomenon can be facilitated provided these conditions are met. In this study, we identified nine facilitating conditions classified into three categories. Some of these are specified as attributes of the artefacts and agile practices, others as attributes of people.

Artefacts-related facilitating conditions Agile teams create artefacts in the course of product development. These artefacts are useful in tracking product progress, providing transparency and prospects for inspection and adaptation to the stakeholders (Schwaber and Sutherland 2011). Some of the common Scrum artefacts are Product backlog, Sprint backlog, Definition of Done (DoD), etc. (Deemer et al. 2012). Attributes of agile artefacts were reported to facilitate self-assignment, such as F1 (appropriate task information), F2 (appropriate task

Table 3 Facilitating Conditions and Constraining Conditions

\begin{tabular}{lll}
\hline \multicolumn{2}{c}{ Facilitating Conditions } & Constraining Conditions \\
\hline \multirow{2}{*}{ Artefacts } & F1: Appropriate task information & C1: Self-assignment for Dependent Tasks \\
& F2: Appropriate task breakdown & \\
& F3: Well-defined Definition of Done & \\
& F4: Well-groomed product backlog & \\
Practices & F5: Collective estimation and task breakdown & C2: Urgent Work \\
& F6: Estimation before prioritization & C3: Tracking work distribution and accountability \\
People & F7: Strong product knowledge & C4: Distance Factor \\
& F8: Good understanding of problem & C5: Manager Intervention \\
& F9: People Behaviour & C6: Inadequate expertise \& resources \\
& & C7: Multiple people interested in similar tasks \\
& & C8: Self-assigning tasks not skilled at \\
& & C9: Self-assignment for new team members
\end{tabular}


breakdown), F3 (well-defined Definition of Done), and F4 (well-groomed product backlog). These are detailed through examples below.

F1: Appropriate task information. Requirements-related work items in agile are generally defined as epics or features (for high-level requirements) and user stories or tasks (for lower level requirements) (Bick et al. 2018). High-level work items are generally allocated to the development teams who break them down into user stories and technical tasks either individually or collectively. Providing enough information on the work items was seen to be of vital importance to effective self-assignment and is identified as the most important facilitating condition as stated by a majority of the participants [P14, P18, P19, P20, P22, P26, P28 - P31, P37, P40-P42]. The team members understand the problem and feel confident to self-assign if sufficient details are provided against the work items. Having comprehensive information not only helps the development team understand the problem and propose solutions but also identifies the task dependencies involved and the impact it makes on other modules. Particularly, this supports the junior team members who are initially hesitant to ask for help. Additionally, with enough details on the tasks, it is quite unlikely that team members will have to go to other team members for getting clarifications and instead rely on themselves. This is accepted by both the managers and the developers as indicated in quotes below.

'It [task] should have enough details, that's the most important thing.' -P22, Developer

'You've got to make sure that you have enough information either in the card or in the explanation so that they (team members) do feel confident with taking on that task.' P14, Technical lead \& Developer

F2: Appropriate task breakdown. Appropriate level of granularity while breaking down tasks is seen to drive the work allocation in the right way. This indicates that it's not just the task's comprehensiveness that makes it understandable to team members, but the way the breakdown is done also adds clarity on it. For example, while defining a form if developers start writing about every field name as a task, most of the time will be taken defining it which is not useful in any way. If the tasks are not broken down appropriately it could lead to ambiguity resulting in assignee's lack of confidence to complete the task on time. A more decent breakdown of tasks facilitates the individuals in making reasonable choices as it makes the tasks clearer, more understandable, and easier to do.

'The key is not to split tasks to such a smaller level so that it becomes very difficult to

allocate. You want granularity but you want a certain level of granularity' - P18, Software Architect

F3: Well-defined Definition of Done. DoD provides clarity to work item's (feature, story, or task) definition and is considered met when it fulfils the customer's acceptance criteria. If the acceptance criteria or $\mathrm{DoD}$ is vague and lacks clarity, then there is a potential risk of wrong interpretations of the work items. The team members may not pick them to avoid discussions required to gain clarity or assume the task could be harder to complete. They may not pick them considering that fleshing out the right acceptance criteria would be an additional task. Well-defined done criteria help in making effective choices while self-assignment tasks, as stated by P27. 
'It is important that done criteria is properly defined at the beginning of the sprint or whenever the task is available, with insufficient DoD they [team members] are unlike to choose the work'-P27, Developer

F4: Well-groomed product backlog. Agile teams perform product backlog grooming and refinement sessions mainly to refine and improve user stories, and to estimate and prioritize the backlog items (Deemer et al. 2012). A well-refined structure in the product backlog seems to contribute as a facilitating factor towards effective self-assignment. The backlog should not be only well-groomed but also consistent so that it's not undergoing extraneous changes in priorities. With too many changing priorities, the backlog can be unwieldy and challenging to manage as indicated by P29.

If you have an environment where the backlog of stories coming up, or switching the priorities, or changing every day, then it's hard' - P29, Developer \& Scrum Master

Well-defined and detailed artefacts and concepts such as the technical tasks or user stories, product backlog and definition of done facilitated self-assignment.

Practices-related facilitating conditions Facilitating conditions consisted of practices such as F5 (collective estimation and task breakdown) and F6 (estimation before prioritization).

F5: Collective estimation and task breakdown entails a combined effort involving everyone in the team (Deemer et al. 2012; Hoda and Murugesan 2016). This helps in getting input from all the team members, sometimes defending their individual estimates, sharing assumptions and knowledge, keeping all on the same page, therefore providing all team members the opportunity to choose any task. This collective estimation and effort support collective awareness of the task. No one can disregard a task as the team members collectively perform the breakdown and estimation of tasks, share the information, help and indicate the right direction so the chances of mistakes and inaccurate estimates can be less.

'During the planning we do everything together, sharing, creating the tasks, it means that everyone knows and owns those tasks. So, no one could say I didn't grab a task, it's not my estimate' - P15, Technical Lead \& Scrum Master

F6: Estimation before prioritization. In a few cases, it is seen as important to estimate tasks well in advance of the sprint. Having estimations a few iterations ahead of the sprint was seen to help the teams practice self-assignment since it ensures a long list of tasks is available to choose from providing more options for the team to select and exercise autonomy. This provides an opportunity to get prepared for the work in advance allowing the team to move tasks as per their and business needs. As a result, team members can commit to tasks of their choice.

'We made sure that we were about 4 to 5, maybe more, Sprints ahead in estimation at any point in time. So the problem with prioritising before estimation is that when the team commits, the set of options is very small so they don't actually feel like they're exercising autonomy. So by giving us the flexibility to be 5-6 Sprints ahead, allowed the team to go, 'you know, if we do this thing that's in Sprint number 4 now, you know, we're preparing the groundwork for something that's coming later, let's move that up'. And now the team starts self-organising or practicing autonomy'-P30, Lead Developer 
As reported, this worked well in an experienced autonomous team of developers who were free to bring items into the backlog, based on their requirements. The team was doing estimations within a two-week Sprint, product grooming three times, every two weeks. It should be noted that estimating 4-5 sprints in advance may not be practical in all settings due to time constraints. However, estimating 2-3 sprints ahead may not be that unrealistic as a trade-off for the team to self-organize and practice autonomy.

People-related facilitating conditions Some attributes of the people involved in the selfassignment, such as F7 (In-depth product knowledge), F8 (Good understanding of problem), F9 (People behaviour including technical self-awareness, sense of ownership, understanding of importance) are also reported to mediate the self-assignment process.

F7: Strong product knowledge. Strong in-depth product knowledge makes developers and testers familiar with different areas of the application. That makes them more competent, and they are more comfortable to make the right choices when self-assigning tasks. It is likely to build their confidence, increase productivity, and improve their work quality.

'Well naturally whoever knows the area of work, the piece of software or the problem that needs to be addressed that's most productive' - P20, Lead Developer

F8: Good understanding of problem. Also, understanding the work items and associated problems plays an important role as acknowledged by both developers and Scrum Masters. With an incorrect understanding of a problem, it is possible that the attempts to resolve the problem will also be flawed. Therefore, having a mutual and accurate understanding of the problem is important for self-assignment. Developers are typically seen reluctant to choose the tasks that they do not understand well as indicated by $\mathrm{P} 29$.

'Having a good understanding of the stories that need to be done, I think that is important. If I have many questions about a story, I can't self-assign, because I don't know what needs to be done.' - P29, Developer \& Scrum Master

F9: People Behaviour. Additionally, other behaviours and attitudes that were reported as facilitating conditions by multiple experienced managers and team members were: selfawareness of technical abilities as a team or as individuals and having sense of ownership and commitment. If the individuals and teams are well-aware of their technical abilities, they would make reasonable choices individually or collectively.

It has been acknowledged both by the managers and agile team members that when people select a task, they have the freedom to choose their own direction which boosts their motivation to perform better.

'The most important thing in my view is people have buy-in, they commit and agree on the tasks that they want to go and do. And I think that gives them a sense of ownership, it gives them a sense of choice and commitment.'-P42, Technical Lead

With this autonomy and opportunity to choose, one can naturally grow responsibility and commitment towards that work enabling a sense of ownership. On the other hand, if the team members are being forced to work on something, they are less likely to own it. This indicates if these attitudes are manifested in individuals, they can help to facilitate the self-assignment process. 


\subsubsection{Conditions Constraining Self-assignment}

We identified ten conditions that were seen to constrain self-assignment through posing some challenges. Similar to the facilitating conditions, these fall under Practices, Artefacts and People-related conditions.

Artefacts-related constraining conditions The only constraining condition reported in this study under artefacts is C1 (Self-assignment for Dependent tasks) which is listed below.

C1: Self-assignment for Dependent tasks. Some tasks rely on other tasks to be completed before they can be started. This can sometimes be challenging as some developers may pick work which may have a dependency on other tasks in the sprint. If the team members are unaware of these dependencies, they will likely self-assign such tasks, which can lead to slow or minimal progress.

'Certain stories are dependent, but we avoid that as much as possible' - P32, Developer

'We try to avoid having dependant tasks, but it happen' - P16, Developer

Practices-related constraining conditions C2 (Urgent Work), C3 (Tracking work distribution and accountability), and C4 (Distance Factor) are identified as constraining conditions influencing the self-assignment process.

C2: Urgent Work. Many participants indicated that urgent work coming during the running sprint is one of the most influential factors that constrains practicing self-assignment [P13, P14, P16, P18, P19, P21, P23, P25, P28, P30, P33-P36, P40, P41]. When there is some high priority urgent task, e.g. a high impact bug in some part of the application or a show-stopper support reported by the customer, then self-assignment is constrained. An example of such work is shared below.

'When product owner is getting feedback from the app stores about.....being annoying for customers...., Well guys, it's really important that we squeeze this in as customers are really complaining about it' $-P 23$, Test Analyst

This is sometimes disturbing for the team members as it supersedes their ability to choose and takes away time and resources from the ongoing sprint. One of the participants disclosed this as follows:

'Obviously, there are urgent stuff that just gets put onto my desk'-P19, Developer

Another participant indicated that they could refuse to take up such urgent things but find it culturally incorrect. This could be because knowing the urgent nature of the work, and still not showing a willingness to work on such task may not please the manager or contradicts the team or business interest.

'Although we can say no, we're not gonna do it, but it wouldn't be culturally nice to say that' - P23, Test Analyst

C3: Tracking work distribution and accountability. Multiple team members choosing the tasks on the go during the running sprint gets challenging as no single individual is directly accountable for any specific issue which is reported later on. This is because multiple people contribute to one story by committing to different tasks. For instance, a story X may consist of 10 tasks, and if these tasks are done by five different developers, it could be hard to backtrack 
an issue as so many developers have been involved in the development of the story as stated by one participant. However, this is not reported to happen frequently.

'You may get [into situations], like if there's a problem found [later], there may be less ownership on, maybe five people worked on a story, well, whose bug is that, yeah (laughter).' - P15, Technical Lead \& Scrum Master

As the team members are given freedom to choose tasks they may not choose wisely and make wrong estimations. The reasons could be that they try to impress a manager by taking more, long or complicated tasks or want to show their efficiency by working harder. This can sometimes lead to situations where the product is delayed due to the fact the person is not able to finish the tasks they committed. They are given a choice, but their wrong choice led to significant delays. However, managers sometime feel that people are not choosing enough tasks for a sprint.

'The only bit of it[self-assignment] that I don't like is it can get a little bit unambitious in terms of what can I get done. Like it's easy to have an expectation set of 20 points per person, per Sprint for example. And mentally that's what I tend to think ... But sometimes I wonder if there would be more that could be done if people worked harder...And I felt like either somebody wasn't working on their tasks or it wasn't getting done' - P31, Development Manager

One the other hand, one of the participants P20 shared the experience of penalizing by overcommitting more tasks in a particular sprint and acknowledged picking amount of work that they are sure to accomplish.

I [team member] remember my took on a lot of work through, and hadn't finished things at the end of the sprint and so things were uncompleted and he [Manager] doesn't like that. So, I felt like trying to work hard is penalized. So, what happens now is I'll do all the work in the sprint, won't take on anything else' - P20, Lead Developer

C4: Distance Factor. The distance factor, or remote location of teams and working across different time zones, seems to influence the application of self-assignment in some way as brought up by a couple of participants. This especially happens when half of the team is sitting close to the Product Owner or the client while the other half don't have Product Owner or the client representative. They don't get as much connectivity as the collocated ones and particularly disadvantaged when people don't speak very clearly during discussions, missing some important piece of information. Similarly, the collocated members get an edge of expressing their interest for any task grabbing it earlier, enjoy the opportunity to show their enthusiasm and collaborate with the client in person. When the development team is collocated, it enhances communication and coordination of activities while picking tasks, e.g. sharing prior knowledge on a task, less or no pair programming with a remote team member. Working with teams in different time zones is more challenging. There is a good chance to struggle to get a task of interest if teams are operating in different time zones.

One of the team members who worked remotely revealed that being away from team physically sometimes jeopardized practicing self-assignment in its true essence.

'Sometimes we are on remote call, client and US team are together in same room, when they start picking the tickets, having discussions, everyone is interested doing that work they have advantage of raising their hands they will quickly say 'Hey, I'm interested 
...they have advantage.... auction never starts here' -P1, Tech Lead

'If some person is on a different time zone, he's still sleeping, and the job comes in today, how can he know, how can he assign himself on that? I'm going to do it.' - P33, Tester

One manager shared how working dynamics such as real physical presence, missing facial expressions and gestures, sharing thoughts and skipping offline talks and different insights can undermine the self-assignment for people working remotely.

'If you're not in the room with seven other people, you're on a speaker phone, you can't see what's going on, don't experience the dynamic. And then people vote because you're not seeing the hands go up, you're not influenced by the democratic process. So, you have a different thought or insight because everybody else has been talking about it offline or whatever the case may be so, there's a gap.' - P30, Lead Developer

While observing one of the stand-up meetings [T11], one developer who used to work remotely for a couple of days every week due to some personal situation seemed disadvantaged. The daily stand-up was a lot harder, he had to dial in for it, and the team had to relocate to the recreation area for making the call. While observing the stand-up, we also noticed that the people weren't speaking very clearly, so he probably did not hear half of it and even his voice broke up once during the call. Above we have included a memo (Fig. 6.) saved in NVivo on to exemplify the influence of distance factor on making self-assignment work.

Some intervening conditions apply to a specific context as identified by memo (See Fig. 5.), e.g. distance factor is specified as one of the constraining factors, but this only applies when one or more team members are working remotely. These constraining conditions lead to certain action/interaction strategies which are adopted by agile individuals and teams as presented in Fig. 7.

People-related constraining conditions Some of these constraining conditions are associated to people's behaviours. These are C5 (Manager Intervention), C6 (Inadequate expertise \& resources), C7 (Multiple people interested in similar tasks), C8 (Self-assigning tasks not skilled at), C9 (Self-assignment for new team members), and C10 (Personality Traits).

C5: Manager Intervention. Some technical managers or leads were often found proposing or suggesting their way of doing things. This emerged as another intervening condition in letting team members practice self-assignment. The managers may not necessarily push their decisions, but team members may not like this interference while performing the task. They rather prefer doing it on their own without any directions as shared by P19.

'But there definitely been times when he [manager] looked over and given suggestions.

So, I don't really mind but I prefer him to not be there just so I can do it [task] on my own.' - P19, Developer

On the other side, manager intervention can also be inadvertent. One manager talked about instances when it's not their intention to assign tasks but the gestures like looking at someone during the daily stand-up, asking a question about a task or discussing an issue gives them an indication that the manager wants them to pick it. Another manager accepted that there are still 


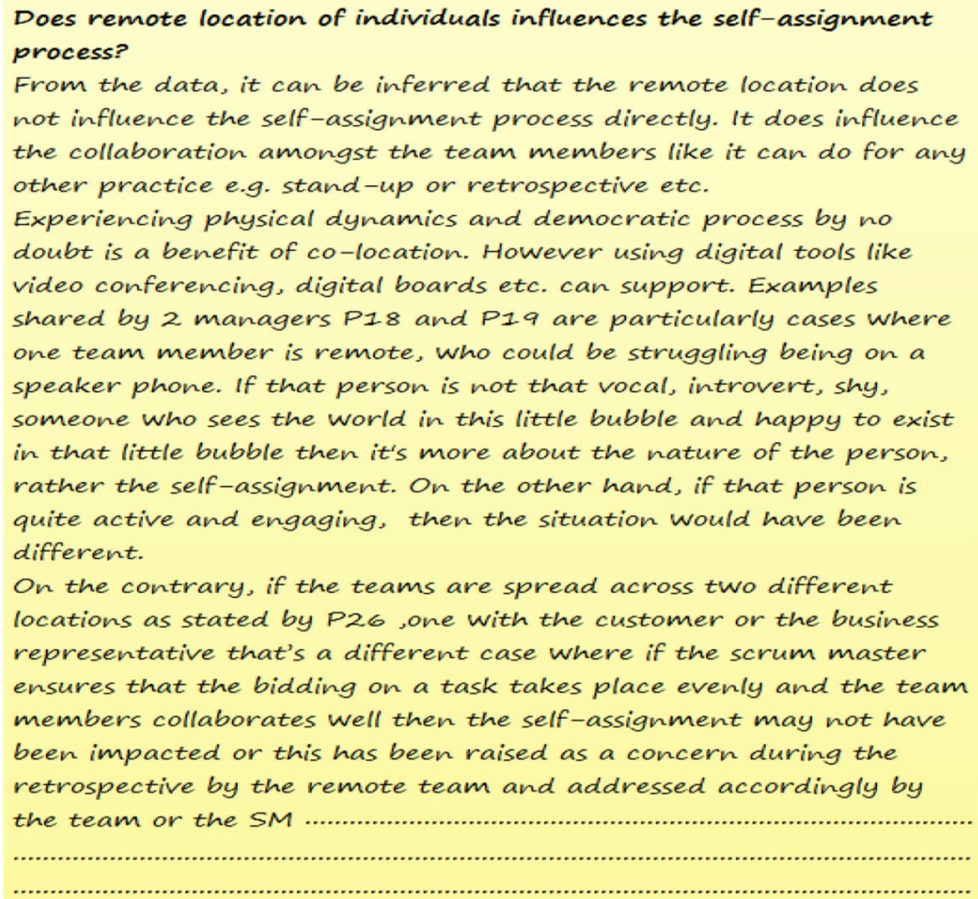

Fig. 6 Memo on influence of distance factor on self-assignment

times when they could not resist assigning a task, limiting the team members to make their own choices.

'I guess there are still times where I might go up to someone and effectively assign them the task, because I've asked them a question and then I've said can you look into this...

So that still does happen.' - P31, Development Manager

Similarly, while observing team's sprint planning meeting, this was also noticed that the manager having an eye contact with one of the developers while elaborating a story might have influenced the developer choosing the story as that team member was seen to self-assign that story.

C6: Inadequate Expertise \& Resources. As another constraining factor, sometimes inadequate or limited resources are seen to influence the smooth execution of self-assignment. As an example, in a team with one tester, there is no option of choosing tasks. As an exceptional case, when most of the members in the team happened to be away, then also self-assignment is kept back.

'There's no self-assignment, because the Quality Assurance Analyst is a single person, he cannot, it's only the Quality Assurance Analyst who can take up the thing -P29, Developer \& Scrum Master

Also, sometimes managers and scrum masters have to assign tasks to keep a balance for equal distribution of work among the resources. For instance, if there is a high priority task that must be assigned, it goes to the person who is free but if it was not high priority, it could just go in the queue. Participant P21 shared an example of this as: 
I [Scrum Master] tend to have something in my mind about who might be assigned partly because I want to make the logistics work, this person becomes free, this person has some other work therefore it probably goes to the person who is free.'-P21, Scrum Master

From these examples, it is evident that sometimes when the resources are not fully available the manager has to purposely suspend self-assignment. Also, to keep a check and balance. This indicates that the availability of expertise and resources also impacts the self-assignment process.

C7: Multiple people interested in similar tasks. There are times when many developers/ testers are interested in picking same tasks. This could be due to the level of ease or interest, potential for outside endorsement, opportunity to learn new technology etc. However, it could sometimes get challenging to not let the same people pick the fascinating ones, keeping an equal balance among all the team members and getting the full benefits of self-assignment.

'As you're [team] working down the board, getting stories done, you know, maybe the one [task] everyone wants to do is story number 4...' -P3, Technical Lead \& Scrum Master

C8: Self-assignment tasks not skilled at. Different instances were revealed around people's reactions as constraining factors towards self-assignment. Developers and testers are seen to choose tasks that they might be interested in doing to explore and learn new things, and this sometimes ends up into low productivity, needing more help or making wrong estimations. This is because they may perceive the level of difficulty and effort required to complete the task incorrectly. The task could be more challenging and time-consuming than initially anticipated. But an encouraging manager has to outweigh these, firstly for the promising benefits of employee satisfaction through some control over what they pick for themselves and secondly allowing them to try, learn and improve their skills. However, this can be challenging as the task may need to be estimated accordingly or given more time for completion. It is also reported that sometime someone picks a task they are not skilled at and struggle later on which is indirectly encountered as another challenge with self-assignment.

'A person might go and take a task that they're not the right person for. So e.g. there might be a very specialist task in a security piece of work, and a person who might not have self-awareness might go and pick it up. And rather than them doing it in an hour, it might take about three days' -P42, Technical Lead

C9: Self-assignment for new team members. Newcomers are neither well-acquainted with their fellow members nor with the team's development processes in the beginning. They require some time to settle in, understand development practices, build trust and co-ordination with other team members. Similarly, introducing new members to self-assignment seems challenging, irrespective of being a novice or experienced professional they need some assistance to understand the team's task assignment process in addition to getting an understanding of the technical domain and code base.

'They're [new member] just starting to know everything [process \& project] and in a complex project as this, if you ask me, I would like them [Manager] to assign as I don't know a thing about it' - P33, Tester 
C10: Personality Traits. Some people struggle in having confidence in their own choices, it might be part of their personality, or the culture they come from or due to lack of selfconfidence. For instance, the shy or introvert members may find it intimidating to self-assign a task. They sit back while others self-assign tasks leaving behind the ones not picked up by others. Then, there are also less-confident members who may have the right skillset and knowledge to perform the task but are scared to raise their voice or are under the impression that other team members may be more capable of performing that task quickly and more efficiently. They have a natural tendency to believe in other opinions more and seen more comfortable with working on tasks assigned by others.

'There are members who don't want to pick something, it's hard for them to step in front of the team and take something, rather than getting something. And that is a personal attitude, and that's hard and if you have a team where more than one is like that, it's hard to counter...' - P32, Developer

\subsection{Actions/interactions Strategies- To Workaround Challenges of Self-assignment}

The constraining conditions described in sub-section 4.4.2 steer the individuals and teams to adopt strategies for overcoming the undesirable effects of the phenomena. We identified 14 strategies, which we describe in this sub-section and are illustrated in Fig. 7.

S1: Task delegation Task delegation is the most common strategy $[N=16]$ used for an urgent piece of work (C2) and when the team is short of resources (C6). Our analysis suggests that very high priority tasks are assigned directly to the person considered best suited, the specialists as indicated by a lead below.

'So typically, I'd pick one of the more specialist people who know what's going on and say 'hey, can you please jump in and grab this task?' - P14, Technical lead \& Developer

Sometimes the task is allocated to the most suitable person with the desired technical skillset, at other times it may be directed to a person who has done similar work in the past as expressed by Participant P21 through an example:

'We made a change in partition manager [module] three months ago, and this is related to that change. 'You did that change, so you understand it. Can you go and do it?' P21, Scrum Master

This can result in a quick solution to the problem but was perceived as a threat to autonomy as the team members are no longer allowed to choose their own tasks, rather the assignment is being enforced on them through their manager.

S2: Offering work An uncommon strategy $[N=6]$ practiced to address urgent work (C2) is that the manager will post a message through online channels, like slack or email, or during the stand-up indicating the high priority of the task and let the team members choose. Listed is an example where true autonomy can be easy to practice by providing the opportunity of choice as a variant of self-assignment in the form of volunteering. 


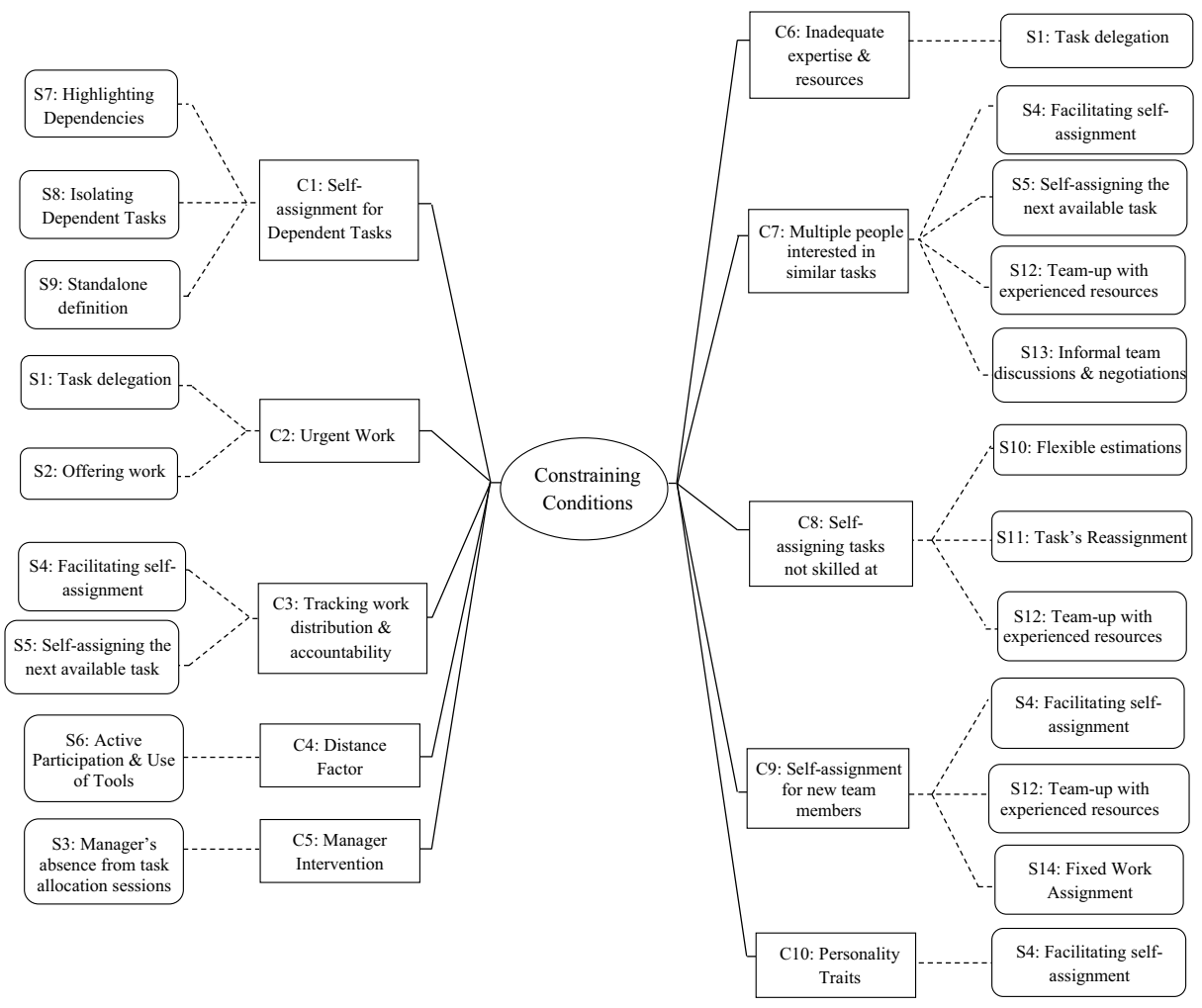

Fig. 7 Action/Interaction Strategies for constraining conditions. The rectangular boxes represent constraining conditions, and the round-cornered boxes represent the strategies. Dashed lines link the constraining conditions with their respective strategies

'X [Manager] posts a message that this ticket is priority, can someone have a look and then everyone will volunteer' - P33, Tester

S3: Manager's absence from task allocation sessions To minimize the influence of the manager (C5), teams are seen to conduct the task allocation sessions without them. It helps them choose their tasks without the manager's persuasion.

'Had to persuade dev manager that [stepping out] would work and worked in other places till he reckoned and agreed the team was a bit more mature and he would step back letting them assign the tasks themselves and do their own breakdown.' $-P 21$, Scrum Master

Managers seem to have this self-realization too as expressed by P31.

I felt that I could be a little bit coercive too by saying yeah, $X$ would be best to work on that one, and then suddenly he's assigned to it by default only because I said that. And so that's why I don't participate in those meetings.' - P31, Development Manager

We observed during a sprint planning meeting [T11], the manager briefed all the user stories to the team, and they collectively estimated them. Then the manager left the meeting room, and the team conducted the task breakdown session without him. 
S4: Facilitating self-assignment The scrum master is seen to play an influential role for ensuring an even distribution of work within the team (C3). When managers believe people are not choosing enough tasks for a sprint, it is the scrum master who is seen investigating the underlying cause. People may not be picking more tasks due to low confidence, no experience, lack of interest, other commitments such as working on other business as usual tasks, or to help others. In exceptional cases, when multiple team members show interest in similar tasks (C7), sometimes it's the scrum master who intervenes to keep a balance ensuring everyone gets equal opportunities to learn and grow by experimenting new things.

Similarly, individuals and teams new to agile practices (C9) sometimes are seen struggling to adopt to that level of self-organization due to multiple reasons such as team member's background, experience and attitude. It was shared by the scrum master [P21] that they started practicing selfassignment only to be part of the project initially i.e. practicing it for new development work. This was done to persuade their technical manager who had concerns around meeting a deadline when client demanded quick completion of work. SMs' shared their experiences, when they had issues trying to get some members to take ownership and operate autonomously. There are diligent members who have no trouble picking tasks voluntarily, while it is also not unusual that there are members who barely self-assign unless everyone else in the team has self-assigned tasks. They rely on the SM to suggest them what tasks to self-assign. In such cases, scrum masters and managers are seen to play a primary role to encourage team members to volunteer and steer the team in the direction of self-organization as indicated below.

'I am trying to get people in the way of thinking more with agile mindset. But also try not to push them too hard or too fast, cos then they kind of resist it'-P29, Developer \& Scrum Master

'We're trying to build a culture where people volunteer for stuff when Sprint planning happens. But we don't have a team that is currently groomed with that attitude and mindset. So, we're coaching them to be at that stage, so we ask them to call themselves out on what they want to work on, because they're unsure of what to pick up first'-P26,

Product Owner

Similarly, a good coaching conversation or one-on-one mentoring by the scrum master is reported as a strategy to help people who are not comfortable in raising their voices and choosing work for themselves $(\mathrm{C} 10)$. However, as indicated by the participant this does not happen straightaway and demands a supportive scrum master and consistent team support to help shy, introverted people make choices and feel confident in their decisions.

I had one colleague, he was very silently, he was not really talking, he was a wonderful developer, he was really, really good, but he was not able to step in front of the team and take something. And I worked very long with him together, and we 'taught' him, and mentored him on a friendly way. It took a while, a long while ......... Because I taught him, I was kind of his mentor ... and he learned it. - P32, Developer

This also goes back to the type of culture the team possesses. In an environment where people can have open discussions and address such problems either on individual or team level, this is easy to address. On an individual level, it is mostly the scrum master, mentor or coach who is responsible to facilitate the self-assignment process providing the guidance and helping them to overcome individual problems towards self-assignment. On the team level, the development team members work together to facilitate self-assignment, e.g. senior peers are also seen to play a significant role to support the junior team members. 
S5: Self-assigning the next available task When many people show interest in the same tasks (C7), for most of the teams the sprint rule of self-assigning the next available task automatically handles such situations. The first person who runs out of work can take the next available task on the storyboard. A senior participant shared that even being a senior developer, if he likes to do a task, at times he misses out because of this rule. This naturally addresses the issues of short of work, unequal distribution, under-committing, and overcommitting of tasks (C3). In this scenario, it is to be ensured that there are enough tasks on the board so that no one gets short of work. It was observed during the sprint planning meeting [T11] that the scrum master included few stories as 'could have' to ensure everyone has work. These were treated as stretch tasks for the sprint.

'As you're[team] working down the board, getting stories done, you know, maybe the one[task] everyone wants to do is story number four, but no one can go to it until story number three has no more tasks they can work on. So, but the first person who runs out of tasks above that story will grab the task.' - P15, Developer \& Scrum Master

S6: Active participation and use of tools Software tools facilitate self-assignment by providing all the information related to a work item in one place. They serve as central source of information and enable teams to stay up to date, increase transparency and visibility of work items. The use of online tools is identified as a useful strategy in keeping the remote members involved during the allocation process (C4). These tools make self-assignment easier as the team members can just access the tool irrespective of their location, look at the product and sprint backlog and self-assign items. The moment a task is selected it reflects the assignee details against the task. It serves as the single source of truth for everyone making the progress visible to people inside and outside the team, highlighting if people are picking up work, how long they are taking to accomplish the tasks or even used as a platform to ask or offer others help. These tools assist the team members to collaborate and communicate actively.

'That's the reason why we've got systems. So, for example, if I decided to work on this task, I'll go into the system, assign that task against my name, and then nobody can take it from there. So, you can't work on a task unless it has been assigned to you' - P37, Head of Product Delivery

The remote team members are expected to engage more than the non-remote members, as they may be missing information and important discussions due to their physical absence. A participant stated that remote members need to participate more actively than the nonremote members.

'So, you know, I always say that if you're remote, you've got to do more work to engage, and the people that are not remote don't care about your remoteness actually' - P30, Lead Developer

S7: Highlighting dependencies Another way to address dependencies between stories or tasks was through highlighting blockers on the story board to notify others that this task has dependency $(\mathrm{C} 1)$.

'We've got these little magnetic red things, we just go and put a blocker on them [dependent tasks], and the team knows why it's a blocker. When the person finishes that 
card, they'll pull it off, and often they'll just pick that card as the next one anyway, just because they've finished it, and it's unblocked.' - P14, Technical lead \& Developer

S8: Isolating dependent tasks The team shared several ways they handle dependent tasks (C1) and some of them are reported to work well. The most effective and common method $[N=14]$ stated to face the challenge of dependent tasks is isolating dependent tasks across sprints. One sprint takes care of first part of dependency while the next handles the other dependent part.

'The way we do it [dependent tasks] is we do identify that this will block this one. Because we're only doing one-week sprints we sometime put the two cards in two different sprints. So, there's immediately like a divorce between sprints, so you say hey we'll do this one and this one, this one and this one, and often that works quite well.' P14, Technical lead \& Developer

S9: Standalone task definition Defining tasks in a way that they are kept mostly independent from the start is another shared strategy to address dependent tasks (C1). For example, defining a task in one step (including front- and back-end) is seen to be practiced instead of segregating them into front-end and back-end tasks which is more likely to increase dependency and cause delays.

'So, its start to finish, like from the front end to the back end. So, we [team] don't have a story where it's just the front end, and a story that's just the back end, so that then becomes a dependency.' - P16, Developer

'When they [team] slice a story or even the tasks, they create tasks that are what we call atomic, and are standalone.' -P42, Technical Lead

S10: Flexible estimations The most common strategy $[N=8]$ that is reported when developers pick tasks, they are not good at $(\mathrm{C} 8)$ is to give more time i.e. over-estimate such tasks. P15 stated how team estimation goes low in such cases below.

'If someone picks [a task] up, and they're not familiar with it, our [team] estimate starts maybe too low. So, we would expect them to meet up that expectation, and say, maybe it was five hours, maybe the guy says it's going to take me eight hours or 10 hours. And once it gets too big, you go, okay, do you need some help on that'-P15, Developer \& Scrum Master

S11: Task's reassignment In a few reported cases, the work gets taken away from the struggling person (C8) and given to others to accomplish the deadline. Team members find this removal from tasks as demotivating, so this is not specified as a preferred action. Others prefer passing on such tasks as indicated below.

'If someone was struggling, they may give it away, but it's never been taken' $-P 15$, Developer \& Scrum Master

'We've had examples where work has been picked up by somebody, and they've had to pass it onto somebody else to do, that happens.' - P23, Test Analyst

Similarly, another participant specified considering task's urgency to decide whether they will provide assistance or take away the task from them. 
'In those instances, two choices; either we put a mentor to work along with him and train him. If it's not time critical, that's what we would prefer doing. If it's time critical, then we just take the task away from him and assign it someone else.' - P37, Head of Product Delivery

S12: Pairing up with experienced resources Teaming up with experienced resources and providing assistance to speed up the completion is also reported when someone has selfassigned a task, they are not good at (C8). This was also observed during the task breakdown session where two developers worked in parallel, one who was the module specialist worked on the development part of the story, while the other new to the module chose to prepare the unit tests for that story. This was how they were pairing up to work outside their expertise. When multiple developers are interested in similar tasks (C7), senior developers providing assistance is reported as another strategy where senior team members play the role of a mentor leading the other developer through completion of that task sharing knowledge.

'Don't just take the work and do it yourself [senior team members], even though it is easier for you, it's good for them [member picking work not good at] teach them to do it' - P29, Developer \& Scrum Master

Similarly, new team members (C9) are seen pairing up to other experienced team members to obtain help. Having assistance from the day they started, is proven to be useful for new team members. This helps to build confidence over the time.

'Just explain to them [new member], you work from the top down, and grab the next task that, that you think you can work on. We'll probably do it, for the first few weeks, we'll probably help him [new member] choose his tasks that might be easier for them to get into. Because they may not really understand what the tasks are. But after that, they'll just grab something.' -P15, Developer/Scrum Master

Pairing up the new team member with some senior developers is also reported to help them learn and fit in the team as indicated by P14.

I kind of buddied them up with one of my more senior dev [developer]. So, I made it very clear with him [Sr. Developer] that he was really responsible for making sure that this developer was up to speed. And because there was a buddy system, like she would always go to him first for some advice, for some help, and it was part of his day to day business that he had to help her'. - P14, Technical lead \& Developer

At stages when the team members are found struggling with tasks, they have selfassigned (C8), some strategies are reported to address these situations. This is apparent in the shorter Sprints where tasks that are not accomplished get automatically noticed, and people start asking about the obstacles and offering help.

S13: Informal team discussions and negotiations Managers shared multiple strategies e.g. involving all members in team discussions to develop mutual understanding and collective ownership for sprint tasks. This way all team members gained insights into the tasks, increasing their understanding from a technical point of view. So, having these conversations allowed them to make well informed self-assignment decisions. Similarly, another strategy is to encourage team members to have open informal 
discussions when multiple members are interested to work on the same task. This way everyone gets the opportunity to speak up if they want to work on that task. Team members are also seen negotiating with each other to work on tasks that interests them but picked by others (C7).

'There's always room for a team member to say, Look! I've seen that you've assigned yourself to this card. Do you mind if I do it, I've got particular skills in this area? That happens, it does happen' - P23, Test Analyst

On the other hand, a couple of participants indicated this has never been a serious concern and most of the time team members are happy with whatever is on the top of the board.

S14: Fixed work assignment One of the participants shared another strategy where they had a role 'the bug manager' in the team for the new team member (C9). The new team member was only responsible to handle all the bugs and ensure the stability of the platform. This way the new member was introduced to various areas of the application which helped them to explore, learn, and expand their knowledge with practice.

When I [new] joined the team... how do you [team] want me to be the bug manager, when I don't know anything about your platform? Oh, it's not, our platform now. So, I was for two weeks the bug manager, and after the two weeks, I knew the platform. P32, Developer

\subsection{Consequences - Of Strategies to make Self-assignment Work}

The aforementioned strategies are used to overcome situations introduced by the constraining conditions and facilitate the process of self-assignment. These adopted action/interaction strategies helped to practice self-assignment positively, but there are also instances when undesired behaviours of practicing self-assignment are reported as negative consequences of adopted strategies. A list of consequences of these strategies, either positive, negative or both, are listed in Table 4. Details on which consequences relate to each strategy are presented in Table 5 with a few examples elaborated below.

Manager's absence from task allocation sessions (S3) The manager may not know the nuts and bolts of a particular task while delegating it. Letting individuals choose takes off the responsibility from the manager allowing them to use their time and energy for other important and useful tasks. The strategy of not having the manager in assignment sessions (S3) results in effective utilization of manager's time (N2+) as they will be able to invest their time on handling bigger problems then deciding which work should be done by whom.

This promotes autonomy (N1+) and increases opportunity to learn, grow and improve (N6+ ). It will provide individuals a chance to work on different tasks irrespective of their skillset supporting more cross-functionality $(\mathrm{N} 7+)$ in the team. Team members can take on tasks outside of their areas of speciality which help them develop different skills offering them an opportunity to learn, grow and improve (N6+) their skills as stated by a developer.

'It gives an opportunity for the individual to work on tasks that they would like to improve their skills on' - P17, Developer 
Table 4 Consequences of strategies for the constraining conditions

\begin{tabular}{lll}
\hline Consequences & Positive $(+)$ & Negative $(-)$ \\
\hline N1: Autonomy & $(+)$ promote & $(-)$ threaten \\
N2 Time utilization & $(+)$ effective & $(-)$ ineffective \\
N3: Team culture & $(+)$ healthy & $(-)$ unhealthy \\
N4: Delivery & $(+)$ quick & $(-)$ delayed \\
N5: Quality & $(+)$ improved & $(-)$ unimproved \\
N6: Opportunity to learn, grow \& improve & $(+)$ increase & $(-)$ decrease \\
N7: Cross-Functionality & $(+)$ promote & $(-)$ threaten \\
N8: Productivity & $(+)$ increase & \\
N9: Team Communication & $(+)$ increase & \\
N10: Knowledge sharing & $(+)$ increase & \\
N11: Transparency & $(+)$ increase & $(+)$ increase \\
N12: Accountability & $(+)$ effective & \\
N13: Self-management & & \\
\hline
\end{tabular}

This improvement is not limited to individual's technical skills, but also provides an opportunity to work on unexplored parts of the product. This technical learning can be more impactful when complemented with extensive product knowledge for career development and growth.

'That way [self-assigning] we start discovering parts of the software that you not familiar with'-P20, Lead Developer

This autonomy helps developers with effective self-management (N13+) and control their tasks. They could manage their own work e.g. prioritizing smaller, easier or harder tasks first suiting their convenience. The time they spend to ask to someone about the next task is utilized increasing productivity $(\mathrm{N} 8+)$.

Task delegation (S1) When an urgent piece of work arrives (C2) or the team is short of resources (C6) and task delegation (S1) is chosen as a strategy, then the manager would want them to work on areas where they would remain focused on their core activities and prior experience as acknowledged below:

'Had it been me [manager] assigning, I would have always gone with my past experience and said, you've done it before, you do it quickly. So, the learning opportunities would have reduced in that kind of a scenario' -P37, Head of Product Delivery

With this task delegation, autonomy (N1-), the opportunity to learn and grow (N6-) will be compromised resulting in threat to cross-functionality (N7-) and healthy team culture (N3-). Furthermore, empowering team members to choose instead of enforcing delegations automatically fosters healthy team culture (N3+) in the long run as indicated by one of the participants.

It [Delegation] will give you some sort of sense of progress in the short term if somebody micromanages other people, I guess you will get some traction and you will get some movement. But I don't think in the long term that is sustainable or beneficial for the type of culture that we want to have' -P42, Technical Lead

But at the same time since the task, in this case, will be done by an experienced person so the chances of errors will be less, the quality (N5+) of the work will be good and the maintenance 
Table 5 Consequences listed against the strategies

\begin{tabular}{|c|c|}
\hline Strategies & Consequences \\
\hline \multirow[t]{7}{*}{ S1: Task delegation } & N1: Autonomy (-) \\
\hline & N2: Time utilization $(+)$ \\
\hline & N4: Delivery $(+)$ \\
\hline & N5: Quality (+) \\
\hline & N6: Opportunity to learn, grow \& improve (-) \\
\hline & N7: Cross-Functionality (-) \\
\hline & N3: Team Culture $(-)$ \\
\hline \multirow[t]{7}{*}{ S2: Volunteering and Offering work } & N1: Autonomy (+) \\
\hline & N2: Time utilization $( \pm)$ \\
\hline & N4: Delivery $( \pm)$ \\
\hline & N5: Quality $( \pm)$ \\
\hline & N6: Opportunity to learn, grow \& improve $( \pm)$ \\
\hline & N7: Cross-Functionality $( \pm)$ \\
\hline & N3: Team Culture $(+)$ \\
\hline \multirow{5}{*}{$\begin{array}{l}\text { S3: Manager's absence from task allocation } \\
\text { sessions }\end{array}$} & N2: Time utilization $(+)$ \\
\hline & N1: Autonomy (+) \\
\hline & N6: Opportunity to learn, grow \& improve $(+)$ \\
\hline & N7: Cross-Functionality $(+)$ \\
\hline & N14: Self-management $(+)$ \\
\hline \multirow[t]{3}{*}{ S4: Facilitating self-assignment } & N12: Accountability (+) \\
\hline & N3: Team culture \& individual well-being $(+)$ \\
\hline & N6: Opportunity to learn, grow \& improve (+) \\
\hline \multirow[t]{6}{*}{ S5: Self-assigning the next available task } & N6: Opportunity to learn, grow \& improve $( \pm)$ \\
\hline & N7: Cross-Functionality $( \pm)$ \\
\hline & N1: Autonomy (+) \\
\hline & N2: Time utilization $( \pm)$ \\
\hline & N4: Delivery $( \pm)$ \\
\hline & N5: Quality $( \pm)$ \\
\hline \multirow[t]{5}{*}{ S6: Active Participation and Use of Tools } & N2: Time utilization $(+)$ \\
\hline & N3: Team culture $(+)$ \\
\hline & N9: Team Communication (+) \\
\hline & N10: Knowledge sharing (+) \\
\hline & N11: Transparency (+) \\
\hline S7: Highlighting Dependencies & N9: Team Communication (+) \\
\hline S8: Isolating Dependent Tasks & N2: Time utilization $(+)$ \\
\hline \multirow[t]{2}{*}{ S9: Standalone definition } & N4: Delivery (+) \\
\hline & N11: Transparency (+) \\
\hline \multirow[t]{3}{*}{ S10: Flexible estimations } & N4: Delivery $(-)$ \\
\hline & N6: Opportunity to learn, grow \& improve $(+)$ \\
\hline & N7: Cross-Functionality (+) \\
\hline \multirow[t]{2}{*}{ S11: Task’s Reassignment (Taken away) } & N3: Taken Away: Team culture \& individual well-being (-) \\
\hline & N4: Delivery $(+)$ \\
\hline \multirow[t]{5}{*}{ S12: Team-up with experienced resources } & N10: Knowledge sharing (+) \\
\hline & N9: Team Communication (+) \\
\hline & N7: Cross-Functionality $(+)$ \\
\hline & N3: Team culture $(+)$ \\
\hline & N6: Opportunity to learn, grow \& improve $(+)$ \\
\hline \multirow[t]{3}{*}{ S13: Informal team discussions and negotiations } & N9: Team Communication (+) \\
\hline & N6: Opportunity to learn, grow \& improve $(+)$ \\
\hline & N7: Cross-Functionality (+) \\
\hline \multirow[t]{2}{*}{ S14: Fixed Work Assignment } & N10: Knowledge sharing (+) \\
\hline & N6: Opportunity to learn, grow \& improve $(+)$ \\
\hline
\end{tabular}


time will not be more (+) compared to a situation where issues could arise due to lack of knowledge or experience. This would get things going quickly (+).

\subsection{Volunteering and Offering Work (S2}

If volunteering and offering work (S2) is chosen as a strategy then it encourages individuals to choose asserting autonomy $(\mathrm{N} 1+)$ which naturally fosters a healthy team culture $(\mathrm{N} 3+)$. However, depending on who picks the tasks, another contextual condition e.g. if an experienced person picks the task this is typically beneficial as the task will be done quickly (N4+) due to previous experience and the quality will not be downgraded (N5+).

'I have experience in this, let me just pick this up and do it, and they can quickly resolve

it. So, we're able to respond quicker to the customer's problems' -P37, Head of Product Delivery

On the other hand, if task is being picked by an unskilled or inexperienced team member, then this can lead to a delay to deliver (N4-) with a potential compromise on quality (N5-).

\section{Discussion}

We found that agile teams are seen practicing self-assignment either as part of achieving selforganization and agile transformation or to address issues with manager-driven assignment, as described in sub-section 4.3. We identified that self-assignment is influenced by a set of intervening conditions i.e. facilitating and constraining conditions which can either facilitate or hinder its adoption as addressed in sub-section 4.4. We also found that different strategies are used to mediate the adoption of self-assignment (sub-section 4.5) with all the ensuing consequences specified in sub-section 4.6. These intervening conditions can also be understood w.r.t. impact they make. e.g. people choosing tasks they are not skilled at is one of the primary challenges, as this leads to delay in delivering but sometimes this is acknowledged as the price for promoting learning and keeping people happy, and is accepted by managers as a trade-off to bear the benefits of self-assignment. However, there needs to be a balance, if all team members choose tasks, they are not skilled at, then this would definitely affect the team's productivity and become a major constraining condition. But if one or two team members, choose tasks outside of their comfort zone that would not make a big difference. So, part of the manager (i.e. scrum master/coach/mentor) role is to ensure that assignments are not leading to failures, imposing risks on the broader context while balancing the need for learning and cross-functionality consistently.

It can also be seen from our data analysis that participants workaround some of the constraining conditions through different strategies. By definition, these strategies are used to 'overcome the undesirable effect of the phenomena'. However, we found that some of the strategies, in fact, are geared towards avoiding self-assignment (e.g. S1, S11, S14) and do not have a positive impact on the team or the process. For example, when urgent work comes in, a major constraining condition, tasks are delegated to the most skilful person as the most obvious strategy which is an underlying threat to autonomy. On the other hand, if the manager asks for volunteers rather than enforcing decisions on them, they will feel they are still making a choice and exercising autonomy, which could give better outcomes. Knowing and understanding the priority and impact of the work, it is generally expected that only experienced or skilful person would be the one choosing such work. Interestingly, most of the strategies (e.g. 
S3, S4, S10) help facilitate and make self-assignment work within their settings. The analysis of data also shows that remote location does not necessarily affect the self-assignment decisions. It may, however, impact communication among the team members like any other agile practices, e.g. remote daily stand-up, retrospective, etc. which can introduce some challenges. Similarly, dependent tasks are specified as one of the constraining conditions, but it may be the poor planning and breakdown of tasks that can cause delays not the selfassignment choices.

The consequences specified in this study can be interpreted as pros and cons of the strategies to practicing self-assignment for individuals, teams and organizations. For instance, the opportunity to learn, grow and improve and self-management can be inferred as individual benefits, healthy team culture as a team benefit and improved quality and fast delivery as organizational benefits arising from the strategies of promoting self-assignment. On the contrary, situations such as taking away a task could influence the well-being of an individual negatively, i.e. demotivate them, delegating a task to a specialist frequently would stall the growth of the other team members, keeping flexible estimates can lead to delayed delivery eventually impacting customer satisfaction and organizational reputation. Our results showed that the scrum masters, technical managers, and team leads play a significant role in mediating these negative consequences to make self-assignment work in a sustainable manner. It would be useful to delve deeper into how the manager or team can mitigate and manage for these negative consequences in future studies. Interestingly, these pros and cons of strategies can also be interpreted as long or short-term consequences depending on the impact they make e.g. within a relatively short period of time, the impact (effective use of manager's time) from not having manager involved in task allocation sessions can be seen. Similarly, delegating tasks might seem to be a fast way of getting the work done, but the impact it makes may not be beneficial for healthy team culture in the long run. On the other hand, outcomes like healthy team culture, improved quality, and better all-round teams may not be achieved instantaneously but will be evident over a period of time.

\subsection{Comparison to Related Work}

Although no other studies dedicatedly addressed self-assignment, there are some related studies addressing benefits and challenges of self-assignment as part of their findings.

Self-assignment helps to keep the teams motivated as identified by one of the empirical studies on agile challenges (Hoda and Murugesan 2016). This has also been supported by our results. Our study also reveals how self-assignment benefits individuals, teams and organizations. Researchers identified some challenges around self-assignment. Poor self-assignment can lead to loss of cross-functionality when the team members pick familiar and simple tasks (Vidgen and Wang 2009; Hoda and Murugesan 2016). However, Scrum master's continuous monitoring and support can help the teams to address the risk of losing cross-functionality (Hoda and Murugesan 2016). Our results also acknowledged that the Scrum master plays a significant role in facilitating self-assignment in agile settings. Some examples include ensuring an even distribution of work with equal opportunities to learn and grow, good coaching conversations providing guidance, and helping team members to overcome individual problems towards self-assignment. Team members avoiding boring tasks (Strode 2016) is identified as another challenge. Team members are often hesitant to pick tasks with unclear requirements and acceptance criteria (Hoda and Murugesan 2016). These were identified as reasons to self-assignment challenges. 
Our study identified other factors that make self-assignment challenging. These are $\mathrm{C} 1$ : Self-assignment for Dependent tasks, C2: Urgent Work, C3: Tracking work distribution and accountability, C4: Distance Factor, C5: Manager Intervention, C6: Inadequate expertise \& resources, C7: Self-assigning tasks not skilled at, C8: Multiple people interested in similar tasks, C9: Self-assignment for new team members, and C10: Personality Traits. In addition, we present a list of strategies such as S1 (Task delegation), S2 (Offering work), S3 (Manager's absence from task allocation sessions), S4 (Facilitating self-assignment), S5 (Self-assigning the next available task), S6 (Active participation and use of tools), S7 (Highlighting dependencies), S8 (Isolating dependent tasks), S9 (Standalone definition), S10 (Flexible estimations), S11 (Task's reassignment), S12 (Team-up with experienced resources), S13 (Informal team discussions and negotiations), and S14 (Fixed work assignment) to overcome these challenges [C1-C10]. It is also pointed out that multiple developers are seen interested in similar tasks due to their individual preferences. We have reported some of these individual preferences as motivational factors developers consider while self-assigning tasks (Masood et al. 2017b) in one of our preliminary study.

\subsection{Implications}

Findings articulated in this study have direct significant implications for researchers and agile practitioners. The main contribution of this research is a theory of making self-assignment work based on rich empirical data. It adds to the limited agile literature on self-assignment and will assist researchers and practitioners in agile community. Other researchers can expand on this research while exploring various aspects of self-assignment and validating the study's theoretical model (Fig. 5) in similar or different settings. This research has implications for agile practitioners. Our descriptions of the positive consequence of self-assignment should encourage novice agile teams and their managers to attempt and engrain self-assignment as a key practice. It will also assist agile teams struggling to practice self-assignment find solutions to their challenges as shared in this study. Our findings can also be used as a guide for the managers to facilitate self-assignment by empowering team members. The theory of making self-assignment work is presented in a form that can be understood and applied through welldefined: (a) context and (b) causal conditions (c) facilitating conditions, (d) a set of constraining conditions (e) strategies applied by agile teams, and (f) a set of consequences to make self-assignment work. Agile practitioners can benefit from these findings in multiple ways. For example, the mapping between constraining conditions and enabling strategies (captured in Fig. 7) can be used to find relevant strategies to tackle the constraints faced by agile practitioners in their unique contexts. For example, they could have flexible estimations (S10) foreseeing any delays. In situations when the assignee struggles to complete the task within the committed time, they should be encouraged to reassign (S11) i.e., pass it on or ask for help without fear or discomfort. The scrum master and the team can mutually decide to help or take away a task considering the task's urgency. As another strategy, teaming up with an experienced member (S12) would help individuals get familiar and speed up the completion time.

From the data analysis and findings of this study, some of the recommendations for managers and teams are presented below. These recommendations are based on the strategies illustrated in the section 4.5 and in some cases one recommendation is related to multiple strategies indicated through the corresponding S\#. 


\subsubsection{Recommendations for Managers and Teams}

- Managers can play a supporting role and encourage team members to choose tasks for themselves to gain benefits of self-assignment (S4).

- Managers can ensure that the self-assignment decisions do not lead to increased specializations or threaten cross-functionality, rather assignment choices provide equal opportunities to learn (technology, applications, and tools) to maintain a balance of knowledge sharing (S4).

- Managers can guide the team members if they feel they have committed to something which is hard to accomplish. However, they should avoid discouraging members to pick complex tasks. Taking away tasks from the team members is also not recommended (S4).

- Once required information has been conveyed among the team and estimates and task breakdown is done, the manager can step out from the assignment sessions (S3).

- If an urgent task comes in during a running sprint, the manager can ask for volunteers rather than imposing tasks on someone. Knowing the time pressure, it is likely that person with relevant skills will pick such a task (S2).

- If multiple people are interested in working on the same task, they can either pair up (S12) or the manager can step-in and let one of them pick a task (S4), ensuring that the next time the other one gets to select their preferred task (S13).

- The manager in collaboration with the team can monitor the status of the tasks on their preferred project management platform, e.g. Trello, JIRA, or a physical Scrum board. For instance, if an assigned task has the same status (e.g. "in progress") for a long time it could be an indication of the assignee struggling to complete that task (S4, S6). Such issues can also be explicitly shared during the daily standup.

- Managers can initially let the new team members observe the team allocating their tasks and understand the task allocation strategies. Other team members can help them choose easier tasks (S4) or pair them up with senior members for better understanding of the process (S12).

- If someone selects a task, they are not familiar with or skilled at, the estimate for the task should take this into consideration and be kept generous to allow for extra effort (S10).

- The team members miss updating their tasks on their preferred project management platform, which can potentially lead to issues e.g. multiple people working on similar tasks. Automatic reminders through tools or reminders in daily stand-ups can be useful to remind them to update their tasks regularly (S6).

- Individuals can pick tasks in the presence of other team members e.g. at planning or at stand-up. This way other team members who have more knowledge about the task can provide assistance and transfer relevant knowledge if needed (S13).

- The manager or team could include some 'stretch tasks' in every sprint, i.e. a few extra tasks ready, elaborated and estimated, in reserve, so team members can self-assign and complete them if they happen to finish all the other tasks early (S5).

- After picking a story or task, if it turns out to be a significant unit of work, then the assignee or the team should break them down into sub tasks and set their status to unassigned on their preferred project management platform so that other members can self-assign them (S11).

- Teams starting with self-assignment can initially apply for a part of their project e.g. new development features, enhancements etc. rather practicing for the entire project (S4). 


\subsection{Evaluation}

We used Strauss and Corbin's criteria list to evaluate the empirical grounding of the study (Strauss and Corbin 1998). We will address these criteria QC1-QC7 one by one. During open coding, we generated the concepts both in-vivo from the practitioners and conceptual codes given by the authors (QC1: Are concepts generated?). Figure 4 shows an example of how these were generated, and the coding process was applied. We systematically defined the relationships between concepts and categories and conceptual linkages applying coding paradigm during axial-coding. The coding processes used in the study resulted in concepts and categories with well-defined properties and dimensions (QC2: Are the concepts systematically related? QC3: Are there many conceptual linkages and are the categories well developed? Do categories have conceptual density?). While the study reports a single central phenomenon (making self-assignment work), it does determine conditions under which the phenomenon happens keeping into account the underlying variations and dimensions (QC4: Is variation built into the theory?). We gathered data from agile practitioners from various companies and different settings to examine concepts in different conditions so that our theory is representative of the contextual variations and wider agile community. We have used the participants' real quotes, anecdotes, and experiences to define the concepts, associated properties and dimensions and have explained with examples (QC5: Are the conditions under which variation can be found built into the study and explained?). We have presented the research methodology (Section 3) and provided the sufficient data (coding examples, interviewers quotes, excerpts from interview guides and pre-interview questionnaire (Fig. 2) and research process details (Section 3) to justify the reliability of the process (QC6: Has process been taken into account?). The authors have explained the study's analysis and findings in corresponding sections and believe the theoretical findings make a significant contribution to the current literature filling the gap with a comprehensive study on self-assignment. The presented theory and particularly the strategies and recommendations are beneficial for the agile practitioners (QC7: Do the theoretical findings seem significant, and to what extent?).

\subsection{Limitations and Threats to Validity}

This study has covered a limited review of related literature in research area as not much is available in literature about self-assignment as a way of task allocation in agile software development. We have not attempted to review the research findings which are not related to agile and software development and acknowledge that as a limitation. Our data set is limited to agile practitioners who showed willingness to participate. We have kept the participants, their companies, products, and third-party clients' data confidential to adhere to the human ethics policy governing this study.

The study includes team practices observations from one company only. The strategies reported by the participants were based on data from phase 2 which involved co-located team members. Few participants shared their past experiences of working in remote settings, or instances where few members worked remotely so it is hard to differentiate strategies more suitable for co-located or distributed teams from the current dataset, this is included as a limitation and potential area for future research. The paper reported the important role the managers play to facilitate self-assignment. However, it is yet to be explored that how managers reconcile individual preferences with team priorities and business goals to make self-assignment beneficial for individuals, teams, and project outcomes. The study did not 
evaluate the effectiveness of the strategies used to work around the constraining conditions, which can be an exciting area for potential future work.

In this section, we describe the validity of the overall research method and findings. The data collected does not represent the entire agile community and we cannot claim generalizability. However, we employed data triangulation through multiple data sources (participants varying in roles, experiences, skillset, context, environment, culture, companies \& domains) on a large dataset to mitigate the threats of lacking generalizability in the study. A detailed description of the data collection methods (pre-interview questionnaires, interviews \& observations), context in which the research was conducted, and the findings are presented in the paper to benefit other researchers who wish to apply these to different contexts and settings. To mitigate the threat to internal validity concerning the author's potential bias towards GT procedures, the coding activities and model representation were discussed and shared for insights with the experienced co-authors throughout the study. We observed the team practicing self-assignment and collected supporting artefacts (e.g. whiteboard images, screenshots from the management tools) from the team to verify the statements made by the team members during the interviews. Additionally, collecting same information from different team members also validated the integrity of the data. We have provided interview quotes as examples to mitigate the reporting bias. To mitigate the risk of possible inadequate description of study constructs, we adopted in-vivo and explanatory descriptive labels for codes, concepts and categories to capture the underlying phenomenon without losing relevant details.

\section{Conclusion}

Self-assignment is not an easy and straightforward practice to follow. In this paper, we demonstrated how self-assignment works in an agile environment. Through interviews with 42 software professionals representing 28 different agile teams from 23 different software companies, and applying the Strauss and Corbin GT procedures, we present the grounded theory of making self-assignment work in agile teams. The theory explains the context and causal conditions that give rise to the need for self-assignment e.g. natural part of agile transformation, issues with manager-driven assignment. It presents a set of facilitating conditions that mediate how self-assignment may be enabled e.g. appropriate task information, collective estimation, and task breakdown. It also presents a set of constraining conditions that mediate how self-assignment may be constrained e.g. urgent work, manager intervention which are overcome by a set of strategies applied by agile teams e.g. manager's absence from task allocation sessions, flexible estimations, facilitating self-assignment. These strategies result in a set of consequences either positive, negative or both. The study also provides a set of recommendations which can be used by agile practitioners to make self-assignment a valuable practice in their settings. While more empirical work is in progress, it is believed that these findings are a first step towards addressing multiple facets of self-assignment in depth within software agile world and provides a platform for further work. Future work would investigate self-assignment from an individual versus manager's perspective, such as exploring the factors software developers consider while self-assigning tasks, trade-offs to reconcile individual preferences with product goals. 
Acknowledgements We would like to thank all the participants for their valuable inputs to this study. This study was conducted under approval from the Human Participants Ethics Committee at the University of Auckland.

Open Access This article is licensed under a Creative Commons Attribution 4.0 International License, which permits use, sharing, adaptation, distribution and reproduction in any medium or format, as long as you give appropriate credit to the original author(s) and the source, provide a link to the Creative Commons licence, and indicate if changes were made. The images or other third party material in this article are included in the article's Creative Commons licence, unless indicated otherwise in a credit line to the material. If material is not included in the article's Creative Commons licence and your intended use is not permitted by statutory regulation or exceeds the permitted use, you will need to obtain permission directly from the copyright holder. To view a copy of this licence, visit http://creativecommons.org/licenses/by/4.0/.

\section{References}

Acuna ST, Juristo N, Moreno AM (2006) Emphasizing human capabilities in software development. IEEE Softw 23(2):94-101

Almeida LH, Pinheiro PR, Albuquerque AB (2011) Applying multi-criteria decision analysis to global software development with scrum project planning. Springer, International Conference on Rough Sets and Knowledge Technology

Andriyani Y, Hoda R, Amor R (2017) Reflection in agile retrospectives. Springer, International Conference on Agile Software Development

Augustine, S (2005). Managing agile projects, Prentice Hall PTR

Augustine S, Payne B, Sencindiver F, Woodcock S (2005) Agile project management: steering from the edges. Commun ACM 48(12):85-89

Beck, K (2005). Chrysler goes to "extremes". Oct, 1998

Beck, K, M Beedle, A Van Bennekum, A Cockburn, W Cunningham, M Fowler, J Grenning, J Highsmith, A Hunt and R Jeffries (2001). "Manifesto for agile software development."

Bick S, Spohrer K, Hoda R, Scheerer A, Heinzl A (2018) Coordination challenges in large-scale software development: a case study of planning misalignment in hybrid settings. IEEE Trans Softw Eng 44(10):932950

Boehm BW (1991) Software risk management: principles and practices. IEEE Softw 8(1):32-41

Carroll, J And D Morris (2015). Agile project management in easy steps, In Easy Steps

Coleman G, O'Connor R (2007) Using grounded theory to understand software process improvement: a study of Irish software product companies. Inf Softw Technol 49(6):654-667

Corbin, J and A Strauss (2008). "Basics of qualitative research: Techniques and procedures for developing grounded theory."

Crowston K, Li Q, Wei K, Eseryel UY, Howison J (2007) Self-organization of teams for free/libre open source software development. Inf Softw Technol 49(6):564-575

Deemer P, Benefield G, Larman C, Vodde B (2012) A lightweight guide to the theory and practice of scrum. Ver 2:2012

Fetterman DM (2019) Ethnography: step-by-step. SAGE Publications

Giardino C, Paternoster N, Unterkalmsteiner M, Gorschek T, Abrahamsson P (2015) Software development in startup companies: the greenfield startup model. IEEE Trans Softw Eng 42(6):585-604

Glaser, B (1978). "Theoretical sensitivity." Advances in the methodology of grounded theory

Guide, A (2001). Project management body of knowledge (pmbok® guide). Project Management Institute

Hayata, T. And J. Han (2011). A hybrid model for IT project with scrum. Service operations, logistics, and informatics (SOLI), 2011 IEEE international conference on, IEEE

Hoda R, Murugesan LK (2016) Multi-level agile project management challenges: a self-organizing team perspective. J Syst Softw 117:245-257

Hoda, R and J Noble (2017). Becoming agile: a grounded theory of agile transitions in practice. Proceedings of the 39th international conference on software engineering, IEEE Press

Hoda R, Noble J, Marshall S (2012) Developing a grounded theory to explain the practices of self-organizing agile teams. Empir Softw Eng 17(6):609-639

Jurison J (1999) Software project management: the manager's view. Communications of the AIS 2(3es):2 
Kalliamvakou, E, D Damian, K Blincoe, L Singer and DM German (2015). Open source-style collaborative development practices in commercial projects using GitHub. Proceedings of the 37 th international conference on software engineering-volume 1, IEEE Press

Kelle, U (2007). "” emergence" vs." forcing" of empirical data? A crucial problem of" grounded theory" reconsidered." historical social research/Historische Sozialforschung. Supplement: 133-156

Lee, E (2010). “push vs. pull in scrum.”. From https://blogs.msdn.microsoft.com/elee/2010/01/21/push-vs-pullin-scrum/

Lee S, Kang S (2016) What situational information would help developers when using a graphical code recommender? J Syst Softw 117:199-217

Lin, J. (2013). Context-aware task allocation for distributed agile team. Proceedings of the 28th IEEE/ACM international conference on automated software engineering, IEEE Press

Mak, DK and PB Kruchten (2006). Task coordination in an agile distributed software development environment. Electrical and Computer Engineering, 2006. CCECE'06. Canadian Conference on, IEEE

Masood, Z, R Hoda and K Blincoe (2017a). Exploring workflow mechanisms and task allocation strategies in agile software teams. International Conference on Agile Software Development, Springer

Masood, Z., R. Hoda and K. Blincoe (2017b). Motivation for self-assignment: factors agile software developers consider Cooperative and human aspects of software engineering (CHASE), 2017 IEEE/ACM 10th international workshop on, IEEE

Masood, Z., R. Hoda and K. Blincoe (2020). Supplementary Material - How Agile Teams Make SelfAssignment Work: A Grounded Theory Study. Figshare. [Online]. https://doi.org/10.17608/k6. auckland.12133494.v8

Nerur S, Mahapatra R, Mangalaraj G (2005) Challenges of migrating to agile methodologies. Commun ACM 48(5):72-78

Pinto JK, Slevin DP (1988) Critical success factors across the project life cycle. Institute, Project Management

Schwaber, K and J Sutherland (2011). "The scrum guide." Scrum Alliance 21

Seidel S, Urquhart C (2016) On emergence and forcing in information systems grounded theory studies: The case of Strauss and Corbin. Enacting Research Methods in Information Systems: Volume 1. Springer, pp 157209

Simão Filho M, Pinheiro PR, Albuquerque AB (2015) Task allocation approaches in distributed agile software development: a quasi-systematic review. Springer, Software Engineering in Intelligent Systems, pp 243-252

Stol, K.-J., P. Ralph and B. Fitzgerald (2016). Grounded theory in software engineering research: a critical review and guidelines. Software engineering (ICSE), 2016 IEEE/ACM 38th international conference on, IEEE

Strauss A, Corbin JM (1990) Basics of qualitative research: grounded theory procedures and techniques. Sage Publications, Inc

Strauss, A and JM Corbin (1998). Basics of qualitative research techniques, Sage publications, Inc

Stray V, Sjøberg DI, Dybå T (2016) The daily stand-up meeting: a grounded theory study. J Syst Softw 114:101124

Stray, V, Moe, NB and Hoda, R (2018). "Autonomous agile teams: challenges and future directions for research." proceedings of the 19th international conference on agile software development

Strode DE (2016) A dependency taxonomy for agile software development projects. Inf Syst Front 18(1):23-46

Stylianou C, Andreou AS (2014) Human resource allocation and scheduling for software project management. Springer, Software Project Management in a Changing World, pp 73-106

Urquhart, C (2012). Grounded theory for qualitative research: a practical guide, Sage

Vidgen R, Wang X (2009) Coevolving systems and the organization of agile software development. Inf Syst Res 20(3):355-376

Williams L, Kessler RR, Cunningham W, Jeffries R (2000) Strengthening the case for pair programming. IEEE Softw 17(4):19-25

Yin, RK (2002). "Applications of case study research second edition (applied social research methods series volume 34)."

Publisher's note Springer Nature remains neutral with regard to jurisdictional claims in published maps and institutional affiliations. 


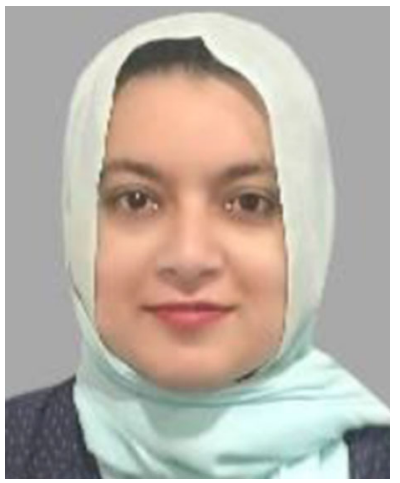

Zainab Masood is currently pursuing her doctoral degree at the University of Auckland (Electrical, Computer, and Software Engineering), New Zealand. Her research interests include agile software development, software testing and quality assurance, and human aspects of software engineering.

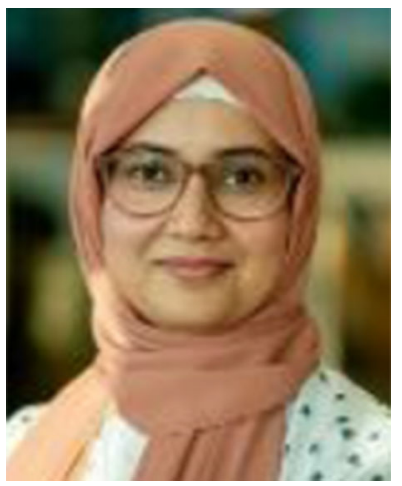

Rashina Hoda , PhD (Victoria University of Wellington), B.Sc. Hons (Louisiana State University), is an Associate Dean (Academic Development) and an Associate Professor in software engineering at the Faculty of Information Technology at Monash University where her research focuses on human-centred software engineering, agile software development, and grounded theory. Rashina received a distinguished paper award at ICSE2017 and a distinguished reviewer award at ICSE2020. She serves on the IEEE TSE reviewer board and IEEE Software advisory panel, as associate editor for JSS and on the organizing committees for ICSE2021, XP2020, and ASE2020. Rashina is currently writing a book on Grounded Theory for Software Engineering. For more information please visit: www.rashina.com 


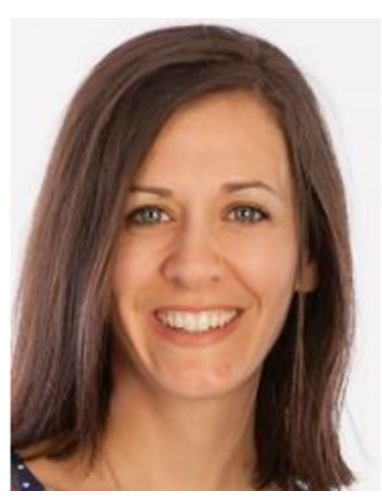

Kelly Blincoe is a Senior Lecturer at the University of Auckland's Department of Electrical, Computer, and Software Engineering. Her research is mainly in the human aspects of software engineering with a focus on collaborative software development and software requirements. She currently serves on the editorial board of the Empirical Software Engineering Journal and the Journal of Systems and Software. She is also on the Executive Board of Software Innovation New Zealand.

\section{Affiliations}

\section{Zainab Masood $^{1} \cdot$ Rashina Hoda $^{2} \cdot$ Kelly Blincoe $^{1}$}

Rashina Hoda

rashina.hoda@monash.edu

Kelly Blincoe

k.blincoe@auckland.ac.nz

1 Department of Electrical, Computer, and Software Engineering, The University of Auckland, Auckland, New Zealand

2 Faculty of Information Technology, Monash University, Melbourne, Australia 